\title{
Effect of Gas-Phase Reaction on the CVD Growth of Graphene
}

\author{
Heng Chen ${ }^{1,3, \dagger}$, Jincan Zhang ${ }^{1,2,3, \dagger}$, Xiaoting Liu ${ }^{1,2,3}$, Zhongfan Liu ${ }^{1,3, *}$ \\ ${ }^{1}$ Centre for Nanochemistry, Beijing National Laboratory for Molecular Sciences, College of Chemistry and Molecular Engineering, \\ Peking University, Beijing 100871, China. \\ ${ }^{2}$ Academy for Advanced Interdisciplinary Studies, Peking University, Beijing 100871, China. \\ ${ }^{3}$ Beijing Graphene Institute (BGI), Beijing 100095, China.
}

\begin{abstract}
Chemical vapor deposition (CVD) is considered as the most promising method for the mass production of high-quality graphene films owing to its fine controllability, uniformity, and scalability. In the past decade, significant efforts have been devoted to exploring new strategies for growing graphene with improved quality. During the high-temperature CVD growth process of graphene, besides the surface reactions, gas-phase reactions play an important role in the growth of graphene, especially for the decomposition of hydrocarbons. However, the effect of gas-phase reactions on the CVD growth of graphene has not been analyzed

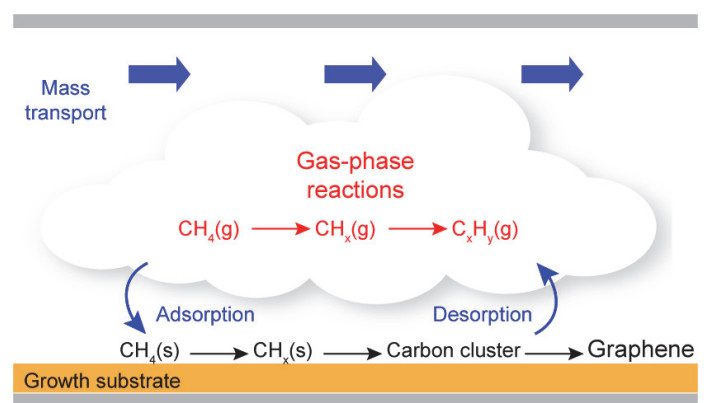
previously. To fill this gap, it is essential to systematically analyze the relationship between gas-phase reactions and the growth of graphene films. In this review article, we aim to provide comprehensive knowledge of the gas-phase reactions occurring in the CVD system during graphene growth and to summarize the typical strategies for improving the quality of graphene by modulating gas-phase reactions. After briefly introducing the elementary steps and basic concept of graphene growth, we focus on the gas-phase dynamics and reactions in the CVD system, which influence the decomposition of hydrocarbons, nucleation of graphene, and lateral growth of graphene nuclei, as well as the merging of adjacent graphene domains. Then, a systematic description of the mass transport process in gas phase is provided, including confirmation of the states of gas flow under different CVD conditions and introduction to the boundary layer, which is crucial for graphene growth. Furthermore, we discuss the possible reaction paths of carbon sources in the gas phase and the corresponding active carbon species existing in the boundary layer, based on which the main impact factors of gas-phase reactions are discussed. Representative strategies for obtaining graphene films with improved quality by modulating gas-phase reactions are summarized. Gas-phase reactions affect the crystallinity, cleanness, domain size, layer number, and growth rate of graphene grown on both metal and non-metal substrates. Therefore, we will separately review the detailed strategies, corresponding mechanisms, key parameters, and latest status regarding the quality improvement of graphene. Finally, a brief summary and proposals for future research are provided. This review can be divided into two parts: (1) gas-phase reactions occurring in the high-temperature CVD system, including the mass transport process and the reaction paths of hydrocarbons; and (2) the synthesis of high-quality graphene film via modulation of the gas-phase reaction, in order to improve the crystallinity, cleanness, domain size, layer number, and growth rate of graphene.
\end{abstract}

Key Words: Graphene film; Chemical vapor deposition; Gas-phase reaction; High quality; Controlled synthesis

Received: January 27, 2021; Revised: February 21, 2021; Accepted: February 22, 2021; Published online: March 1, 2021.

*Corresponding author. Email: zfliu@pku.edu.cn.

†These authors contributed equally to this work.

The project was supported by the National Key Basic Research Program of China (2016YFA0200103, 2018YFA0703502), the National Natural Science Foundation of China (51520105003, 52072042), Beijing National Laboratory for Molecular Sciences (BNLMS-CXTD-202001), and the Beijing Municipal Science and Technology Planning Project (Z18110300480001, Z18110300480002).

国家重点基础研究发展规划项目(2016YFA0200103, 2018YFA0703502), 国家自然科学基金(51520105003, 52072042), 北京分子科学国家研究中心 (BNLMS-CXTD-202001)和北京市科学技术委员会(Z18110300480001, Z18110300480002)资助

(C) Editorial office of Acta Physico-Chimica Sinica 


\section{气相反应对 CVD 生长石墨烯的影响}

陈恒 1,3,十，张金灿 1,2,3,十，刘晓婷 1,2,3，刘忠范 $1,3,{ }^{*}$

1 北京大学纳米化学研究中心, 北京分子科学国家研究中心, 北京大学化学与分子工程学院, 北京 100871

2 北京大学前沿交叉研究院, 北京 100871

3 北京石墨烯研究院，北京 100095

摘要: 化学气相沉积法(CVD)制备的石墨烯薄膜具有质量高、均匀性好、层数可控且可放大等优点, 近年来受到了学术 界和工业界的广泛关注。在高温CVD生长过程中, 除祄底表面的反应外, 气相反应同样会影响石墨烯的生长行为和薄膜 质量。本文将综述气相反应对CVD生长石墨烯的影响: 首先对CVD体系内的气相传质过程和气相反应进行了详细讨论; 随后系统介绍了基于气相调控提高石墨烯的结晶性、洁净度、畴区尺寸、层数和生长速度的相关策略及其机理; 最后对 气相反应影响CVD生长石墨烯的规律进行总结, 并展望了未来可能的发展方向。

关键词: 石墨烯薄膜; 化学气相沉积; 气相反应; 高品质; 可控制备 中图分类号: 0647

\section{1 引言}

石墨烯, 是由单层碳原子以 $s p^{2}$ 杂化形式紧密 堆积成蜂窝状晶格结构的二维纳米碳材料, 拥有 诸多优异的性质, 如高载流子迁移率 (200000 $\mathrm{cm}^{2} \cdot \mathrm{V}^{-1} \cdot \mathrm{s}^{-1}$, 室温 $)^{1}$ 、高热导率 $\left(5300 \mathrm{~W} \cdot \mathrm{m}^{-1} \cdot \mathrm{K}^{-1}\right)^{2}$ 、 高透光性 $(97.7 \%)^{3}$ 、高机械强度 $(130 \mathrm{GPa})^{4}$ 、高比 表面积 $\left(2630 \mathrm{~m}^{2} \cdot \mathrm{g}^{-1}\right)$ 和独特的篎分特性 6 等, 在柔 性可穿戴器件 7 、光电探测与传感器件 8,9 、高效率 散热器件 ${ }^{10,11}$ 、能源存储 ${ }^{12}$ 等领域展现出了广阔的 应用前景。

2004年是石墨烯研究热潮爆发的元年。在这 一年, 英国曼彻斯特大学的Andre Geim教授和他 的学生Konstantin Novoselov用胶带剥离法成功地 从块状石墨中剥离出石墨烯, 并发现了石墨烯独 特的电学特性 ${ }^{13}$ 。六年后, 诺贝尔物理学奖授予给 了这两位科学家, 以奖励他们“对二维材料石墨烯 的开拓性研究”。随后的十几年间, 石墨烯的相关 研究突飞猛进, 而这离不开石墨烯制备技术的突 破性进展。目前, 石墨烯的制备方法主要有机械剥 离法 ${ }^{14}$, 化学剥离法 ${ }^{15}, \mathrm{SiC}$ 外延生长法 ${ }^{16}$, 氧化还 原法 ${ }^{17}$, 化学气相沉积法 (chemical vapor deposition, CVD) ${ }^{18}$ 和快速焦耳热法 ${ }^{19}$ 等。其中, CVD法制备的石墨烯薄膜具有质量高、均匀性好、

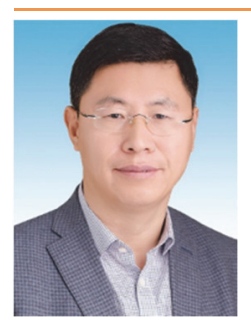

刘忠范, 1962年出生。1990年获东京大 学博士学位。现为北京大学教授, 博士 生导师, 北京石墨烯研究院院长, 中国 科学院院士。主要研究方向为石墨烯的 $\mathrm{CVD}$ 生长方法与应用。
层数可控且可放大等优点, 受到了学术界和工业 界的广泛关注 ${ }^{20}$ 。作为半导体行业广泛使用的一种 薄膜制备方法 ${ }^{21}, \mathrm{CVD}$ 法也被认为是最适合高品质 石墨烯薄膜批量制备的选择。

$\mathrm{CVD}$ 是指利用气态或蒸气态的物质在气相或 气固界面上发生反应, 生成固态沉积物的过程。石 墨烯薄膜的CVD制备通常需要一台高温炉, 在约 $1000{ }^{\circ} \mathrm{C}$ 的高温条件下, 甲烷等含碳前驱体作为碳 源通入高温腔室后, 便会在生长祄底上发生碳源 裂解、活性碳物种表面扩散、石墨烯成核生长等过 程, 最后在衬底表面沉积上一层石墨烯薄膜。研究 者们在不同祄底上制备石墨烯, 并对其生长行为 进行了广泛而细致的研究 ${ }^{22-29}$ 。其中, 铜是制备高 品质石墨烯薄膜最常用的金属祄底之一 ${ }^{20,30}$ 。2009 年, Rodney S. Ruoff课题组 ${ }^{18}$ 首次利用CVD法在铜 箔上成功制备出了大面积石墨烯薄膜, 从此开辟 了高品质石墨烯薄膜材料规模化制备的道路。随 后的十余年里, 研究者们在石墨烯薄膜的结晶性 ${ }^{31}$ 、 表面洁净度 ${ }^{32}$ 、层数 ${ }^{33}$ 、畴区尺寸 ${ }^{34}$ 和生长速度 ${ }^{35}$ 等 的调控方面不断取得新进展, 为高品质石墨烯薄 膜的批量制备和应用探索打下了坚实的基础。

在高温CVD体系内, 石墨烯在祄底表面的生 长伴随着一系列的表面反应。与此同时, 气相中也 发生着复杂的气相反应和传质过程 36,37 。早期, 人 们主要关注祄底表面的反应对石墨烯生长行为的 影响, 并基于此开展了系统的研究工作 ${ }^{26,38-42}$, 而 对石墨烯 $\mathrm{CVD}$ 生长过程中气相反应的影响讨论较 少, 相关工作缺少系统梳理。为填补这一空白, 本 文将系统地综述气相反应对 CVD生长石墨烯的影 响, 在简单介绍石墨烯在金属祄底和绝缘祄底表 
面生长的基元步骤后, 将详细讨论 CVD体系内的 气相传质过程和气相反应; 之后系统梳理气相反 应对石墨烯薄膜的结晶性、洁净度、畴区尺寸、层 数可控性和生长速度的影响, 并列举气相调控制 备高品质石墨烯薄膜的典型策略, 最后总结了气 相反应对石墨烯生长的影响规律并展望了CVD生 长高品质石墨烯薄膜亟待解决的挑战。

\section{2 石墨烯生长的基本概念}

一般来说, 祄底表面石墨烯薄膜的生长可以 概括为碳源裂解、石墨烯成核和石墨烯长大三个 阶段: 碳源进入CVD体系后, 会发生脱氢裂解, 形 成活性碳物种; 活性碳物种在祄底表面迁移、团聚 形成碳团簇，碳团簇会不断捕获周围更多的活性 碳物种, 形成稳定的石墨烯核; 随后, 活性碳物种 在祄底表面不断迁移到石墨烯畴区的生长前沿, 为 石墨烯畴区的长大和拼接成膜源源不断地提供原 料。与此同时, 在石墨烯生长过程中, 气相和祄底 表面存在着复杂且快速的物种动态迁移平衡, 如 活性碳物种的吸脱附过程等, 这也会影响到石墨 烯薄膜的生长行为(图1) ${ }^{43}$ 。下面将对金属和绝缘 衬底表面石墨烯生长的基元步骤分别展开介绍, 对其中涉及到的关键影响因素进行说明, 并在此 过程中厘清气相反应在石墨烯生长过程中发挥的 作用。

对于金属祄底上石墨烯的生长, 碳源的裂解 以金属祄底表面的催化裂解为主 ${ }^{44}$, 同时气相中也 会发生碳源的热裂解和催化裂解。金属类型对碳 源催化裂解的反应势垒和裂解产物影响很大 ${ }^{45}$ 。以 生长石墨烯的主流金属祄底铜和镍为例, 甲烷在 镍表面可完全脱氢形成碳原子, 而在铜表面上很 难完全脱氢, 得到的活性碳物种主要是 $\mathrm{CH}$ 自由
基 ${ }^{46}$ 。随后, 碳源裂解后产生的活性碳物种会在祄 底表面迁移、碰撞, 形成尺寸更大、包含更多碳原 子的碳团簇; 祄底表面形成的活性碳物种也会大 量脱附到气相中, 并在气相中扩散、碰撞形成碳团 簇。这些活性碳物种和碳团簇也有可能再次吸附 到金属祄底表面, 继续参与石墨烯的成核和生长。 石墨烯的成核是指祄底表面碳团簇不断长大的过 程 ${ }^{47}$, 受到成核势垒 ${ }^{48}$ 和活性碳物种含量 ${ }^{49}$ 的影响, 成核势垒越低, 活性碳物种含量越高, 石墨烯的成 核速度越快, 成核密度也越高。比如, 金属祄底的 台阶处相较于平台处的成核势垒更低, 石墨烯更 易成核 ${ }^{48}$ 。石墨烯长大往往伴随着旧键的断裂和新 键的形成, 由于石墨烯畴区边缘碳原子的悬挂键 通常会被氢原子或金属终止 ${ }^{50}$, 终止态的不同会影 响石墨烯生长的反应势垒, 进而影响其生长速度 和形貌。石墨烯的生长速度同样受到活性碳物种 供给速度的影响 ${ }^{51,52}$ 。石墨烯的生长模式有扩散限 制 (diffusion-limited growth) 和生 长限制 (attachment-limited growth)两种 53 。当祄底表面活 性碳物种浓度高, 浓度梯度小时, 石墨烯的生长模 式主要表现为生长限制; 反之, 则为扩散限制。随 着石墨烯畴区的不断长大, 未被石墨烯覆盖的具 有催化活性的金属祄底的面积不断减小, 这就导 致祄底表面催化裂解产生的活性碳物种的浓度不 断降低, 石墨烯的生长速度越来越慢, 甚至无法完 全拼接成膜 ${ }^{51}$ 。此时, 活性碳物种的有效供给成为 石墨烯生长的限速步。通过增加气相中活性碳物 种的含量, 并利用气相与祄底表面频繁的物种交 换过程对石墨烯生长过程中所需的活性碳物种进 行源源不断地供给, 是实现石墨烯的快速拼接成 膜的有效策略之一 ${ }^{52}$ 。因此, 气相中同样发生碳源 裂解过程, 并且气相物种会对金属祄底表面的石

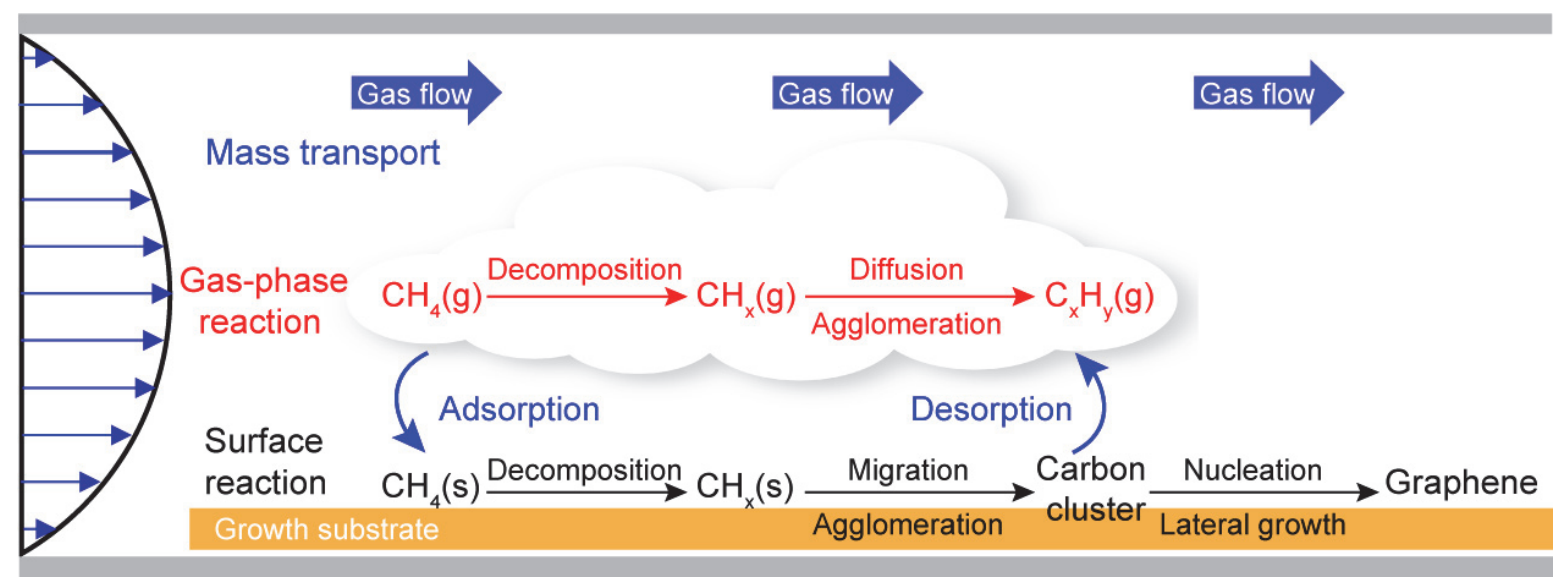

图 1 石墨烯 CVD 生长的基元步骤示意图

Fig. 1 Schematic of the elementary steps in the CVD growth of graphene. 
墨烯成核和长大过程带来不容忽视的影响。

对于绝缘祄底上石墨烯的生长, 尽管基元步 骤与在金属祄底表面基本一致, 但在生长行为上 表现出了明显的差异性。具体来说, 由于绝缘祄底 对碳源的催化裂解作用十分有限, 热裂解是 CVD 体系中碳源裂解的主要方式 ${ }^{54}$ 。由于碳源热裂解的 势垒高, 效率低, 即使热裂解在祄底表面和气相中 同时发生, 祄底表面活性碳物种的浓度仍然极低 ${ }^{55}$ 。 与此同时, 相比于金属表面, 非晶态的绝缘祄底表 面更加粗糙, 缺陷密度更大 ${ }^{56}$; 活性碳物种在绝缘 祄底表面的迁移势垒也远高于金属表面的迁移势 垒 $^{57}$ 。这就导致绝缘祄底表面石墨烯的成核密度更 高, 成核时间更长, 生长速度更慢, 拼接成膜也更 为困难。因此, 相比于金属衬底, 气相中活性碳物 种的供给对绝缘祄底表面石墨烯生长发挥着更加 重要的作用。

简而言之, 由于气相与祄底之间频繁的质量 传输和物种交换过程, 气相反应会影响祄底表面 活性碳物种的类型和含量, 进而改变石墨烯的成 核、长大和拼接成膜的行为, 并最终影响石墨烯的 结晶质量、畴区尺寸、层数均匀性、表面洁净度以 及生长速度等。下面将分别从流体动力学和热力 学的角度对 CVD体系内的气相传质过程和气相反 应进行介绍, 引出对于石墨烯生长至关重要的“边 界层” 的概念, 并分析不同CVD反应条件下气体的 流动状态、气相中的反应类型和物种组成等对石 墨烯生长行为的影响。

\section{1 气相传质过程}

在 CVD体系中, 气体的流动遵循基本的流体 力学规律。具体来说, 可以根据克努森数(Knudsen number, 记为 $\left.K_{\mathrm{n}}\right)$ 的不同将气体的流动状态分为三 种(图 $2 \mathrm{a}$ ): 当 $K_{\mathrm{n}}<0.1$ 时, 气体处于粘滞流状态, 以 连续状态输运; 当 $0.1<K_{\mathrm{n}}<10$ 时, 气体为分子流粘滞流的混合运动状态; 当 $K_{\mathrm{n}}>10$ 时, 气体处于 自由的分子流状态，气体分子间会发生频繁碰撞 ${ }^{58}$ 。 克努森数 $K_{\mathrm{n}}$ 可通过计算分子的平均自由程 $\lambda$ 和特 征长度 $L$ 的比值得到, 如式(1)所示:

$$
K_{\mathrm{n}}=\lambda / L
$$

其中, 平均自由程 $\lambda$ 是用于判断气相中气体分子扩 散难易程度的重要物理量, 可由式(2)计算得出:

$$
\lambda=k_{\mathrm{B}} T / 2^{-1 / 2} \pi d^{2} p
$$

其中, $k_{\mathrm{B}}$ 是玻尔兹曼常数, $T 、 d 、 p$ 分别是体系的 温度、气体分子的平均直径和体系的压力。

在实际的 CVD体系中, 特征长度 $L$ 可以等价为 石英管径, 一般为 $\mathrm{dm}$ 量级。在常规 CVD生长石墨 烯的温度 $\left(1000 \pm 50^{\circ} \mathrm{C}\right)$ 和压强 $\left(10-10^{5} \mathrm{~Pa}\right)$ 范围内, 气体分子和活性碳物种平均自由程最大为毫米量 级, $K_{\mathrm{n}}$ 始终小于 0.1 。此时, CVD体系内气体的流 动状态为粘滞流。此外, 当体系的反应温度、压力 等参数不变时, 也可以通过改变特征长度 $L$ 来改变 $K_{\mathrm{n}}$ 。例如, 可以通过设计铜 “信封” 59 、铜䇴垂直 堆垛结构 60 等方式构建限域空间(图 $2 b)$, 将特征长 度从石英管径变为限域空间的尺寸, 实现粘滞流 到分子流的转变。 (a)

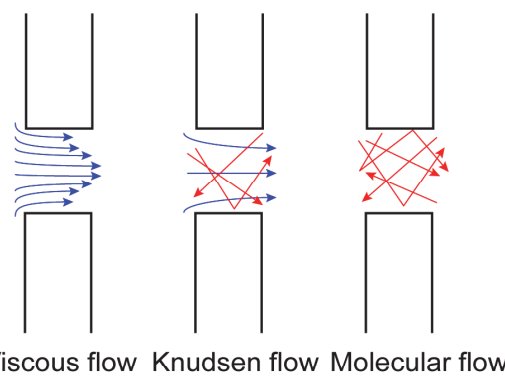

(c)

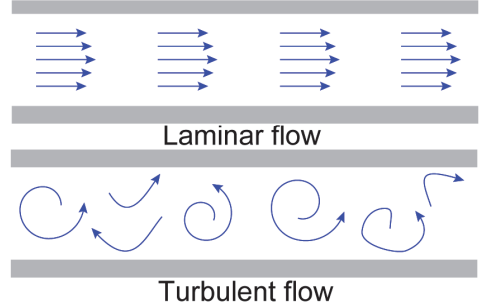

(b)

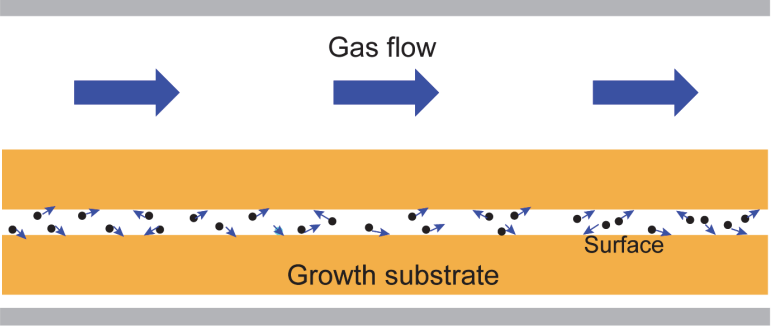

(d)

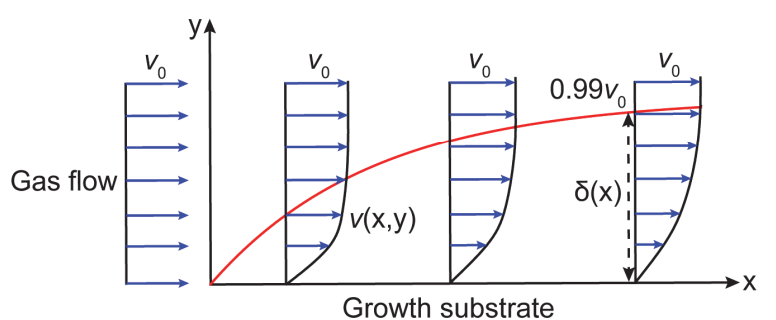

图 2 石墨烯 CVD 生长过程中的气相传质

Fig. 2 Mass transport in the gas phase during the CVD growth of graphene.

(a) Schematic of the three flow regimes; (b) Molecular flow in the confined space based on the special stack configuration of growth substrates; (c) Schematic of laminar flow (top) and turbulent flow (bottom); (d) Schematic of the formation of boundary layer during the CVD growth of graphene. 
粘滞流又可细分为层流、湍流和湍流-层流过 渡的流动状态(图2c)。其中，层流是指流体各质点 沿着轴向平行的方向做互不干扰的直线运动, 而 湍流是流体不仅沿轴向方向做横向运动, 而且沿 着各个方向都会做杂乱无章的运动。雷诺数 $R_{\mathrm{e}}$ 是 判定上述流体状态的依据: 当 $R_{\mathrm{e}}<2000$ 时, 流体运 动状态为层流; 当 $2000<R_{\mathrm{e}}<4000$ 时, 流体运动 状态为层流和湍流的过渡状态; 当 $R_{\mathrm{e}}>4000$ 时, 流 体运动状态为湍流 ${ }^{58}$ 。雷诺数 $R_{\mathrm{e}}$ 可根据式(3)计算得 到:

$$
R_{\mathrm{e}}=\rho v L / \mu
$$

其中， $\rho 、 v 、 \mu$ 分别为流体的密度、流体速度和粘 性常数, $L$ 为特征长度。流体速度 $v$ 可以通过式(4) 代入流体流量 $\Phi$ 求得:

$$
v=\Phi / 4 \pi L^{2}
$$

由公式(5)可求得流体的雷诺数 $R_{\mathrm{e}}$ :

$$
R_{\mathrm{e}}=\rho \Phi / 4 \pi L \mu
$$

理想状态下的层流，气体在不同位置的流动 速率完全相同。然而当气体分子在有限空间内沿 轴向平行方向向前流动时, 贴近容器壁表面的气 体会完全润湿, 流动速率降为零。由于祄底表面的 气体与相邻气体层间存在相对运动, 两者产生的 摩擦使得相邻气体层的流动速率减小。这种由层 间摩擦导致的减速作用随着远离祄底表面而逐渐 减弱, 即气体流动速率逐渐增大。1904年, 德国科 学家普朗特首次提出 “边界层” 的概念, 用来描述 大雷诺数粘滞流中紧贴物面的粘性力不可忽略的 流动薄层。其中, 边界层厚度 $\delta$ 的定义为从有限空 间的边界开始, 沿其法线方向与气体速率达到主 体速率的 $99 \%$ 时所对应的位置之间的距离(图2d)。 由式(6)可知, 随着气体沿着管壁向前流动 $x$ 的距 离, 边界层的厚度 $\delta(x)$ 逐渐增加 ${ }^{20}$ 。

$$
\delta(x) / x=4.64 / R_{\mathrm{e}}{ }^{0.5}
$$

对于石墨烯的 CVD生长过程, 气体进入石英 管前, 可视为匀速运动; 初入石英管, 气体会与石 英管的上下管壁发生摩擦, 向着石英管中心产生 速度梯度; 随着气体向前推进, 边界层的厚度逐渐 增加, 在距管口距离为 $x_{0}$ 的位置, 上下两个边界层 在石英管中心会合, 此时边界层的厚度达到整个 石英管直径的一半。 $x_{0}$ 的数值一般较小。例如, 在 $1000{ }^{\circ} \mathrm{C}$ 的低压 $(100 \mathrm{~Pa}) \mathrm{CVD}$ 体系里, 向管径为 10 $\mathrm{cm}$ 的石英管内通入 $1 \mathrm{sccm}$ (standard cubic centimeter per minute) 的 $\mathrm{CH}_{4}$ 作为碳源和 $100 \mathrm{sccm}$ 的 $\mathrm{H}_{2}$ 作为载气。采用气体的平均半径为 $0.4 \mathrm{~nm}$, 对 应流量的氢气和甲烷的混合气体密度 $\rho$ 为 0.06 $\mathrm{g} \cdot \mathrm{cm}^{-3}$, 气体的粘性常数 $\mu$ 为 $4 \times 10^{-5} \mathrm{~N} \cdot \mathrm{s} \cdot \mathrm{m}^{-2}$ 作为
参考数据进行计算 ${ }^{58}$, 由式(5)可得对应的 $R_{\mathrm{e}}=2<<$ 2000 , 将得到的 $R_{\mathrm{e}}$ 值带入式(6), 可得 $x_{0}=1.5 \mathrm{~cm}$ 。 因此, CVD生长石墨烯时, 衬底表面边界层的厚度 很快达到石英管径的一半, 这就使得气相物种主 要分布在边界层内。

需要指出的是, 边界层的存在对于石墨烯的 生长至关重要, 这是因为: (1)气态碳源和载气到 达祄底表面发生催化裂解反应之前, 必须穿过边 界层; (2)祄底表面产生的活性物种(如 $\mathrm{H} 、 \mathrm{CH}_{3}$ )从 祄底表面脱附, 也需通过边界层扩散出去。在这个 过程中, 边界层内会发生复杂的气相反应, 产生丰 富的活性碳物种, 给气相和祄底表面间频繁而快 速的物种交换和质量输运过程带来影响, 进而影 响祄底表面的石墨烯生长行为。

边界层的存在使得石墨烯的生长由两个基元 过程控制 ${ }^{36}$ : 一是气相中的碳源或活性碳物种向祄 底表面扩散、发生吸附作用的过程, 在该传质过程 中, 穿越边界层到达祄底表面的流量可用式(7)描 述:

$$
F_{\text {mass transport }}=h_{\mathrm{g}}\left(C_{\mathrm{g}}-C_{\mathrm{s}}\right)
$$

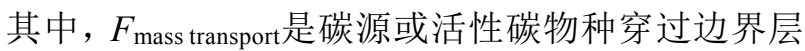
的质量流量, $C_{\mathrm{g}}$ 和 $C_{\mathrm{s}}$ 分别代表碳源或活性碳物种 在气相和衬底表面的浓度, $h_{\mathrm{g}}$ 是传质系数, 由气体 本身的扩散能力和边界层的厚度共同控制。

二是抵达金属祄底表面的活性碳物种经过表 面迁移、碰撞、继而反应生成石墨烯的过程。该表 面反应过程消耗的活性碳物种的流量可用式(8)描 述:

$$
F_{\text {surface reaction }}=K_{\mathrm{s}} \cdot C_{\mathrm{s}}
$$

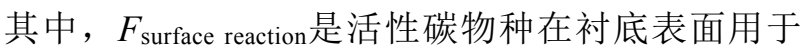
生长石墨烯的质量流量, $K_{\mathrm{s}}$ 是表面反应常数, 与祄 底表面温度有关。

当反应达到平衡时, $F_{\text {mass transport }}=F_{\text {surface reaction }}=$ $F_{\text {total flux }} F_{\text {toatal flux }}$, 即活性碳物种总的质量流量, 可由式(9)描述:

$$
F_{\text {toatal flux }}=K_{\mathrm{s}} \cdot h_{\mathrm{g}} \cdot C_{\mathrm{g}} /\left(K_{\mathrm{s}}+h_{\mathrm{g}}\right)
$$

因此, 当 $h_{\mathrm{g}}>>K_{\mathrm{s}}$ 时, $F=K_{\mathrm{s}} \cdot C_{\mathrm{g}}$, 表面反应过 程成为限制石墨烯生长速度的关键因素; 当 $h_{\mathrm{g}}<<$ $K_{\mathrm{s}}$ 时, $F=h_{\mathrm{g}} \cdot C_{\mathrm{g}}$, 传质过程成为限制石墨烯生长速 度的关键因素 36 。

不同祄底表面石墨烯生长的限速步骤有所不 同。在金属祄底上生长石墨烯时，通常使用低压 CVD体系, 体系反应腔内气体分子浓度较低, 分子 的平均自由程较大, 传质系数 $h_{\mathrm{g}}$ 大于表面反应常 数 $K_{\mathrm{s}}$, 因此, 低压 $\mathrm{CVD}$ 体系通常是表面生长限制过 程; 而使用绝缘祄底生长石墨烯时, 通常采用常压 
CVD体系, 体系反应腔内气体分子浓度很高, 分子 之间的碰撞概率大大增加, 传质系数 $h_{\mathrm{g}}$ 远小于表 面反应常数 $K_{\mathrm{s}}$, 因此, 常压 CVD体系通常是传质限 制过程 53 。

\section{2 气相反应过程}

气相中活性碳物种的来源主要有两部分: 碳源进入CVD腔室后, 直接在气相中裂解形成活 性碳物种; (2)碳源在祄底表面裂解后生成的活性 碳物种从祄底表面脱附后进入边界层。进一步地, 这些活性碳物种也可能会在气相中扩散、碰撞并 通过加聚和脱氢反应形成较大的碳团簇 ${ }^{61}$, 进一步 增加气相物种的多样性和气相反应的复杂性。

以 CVD体系常用的碳源甲烷为例, 动力学模 拟结果表明, 气相中甲烷消耗的主要路径是脱氢 反应生成 $\mathrm{CH}_{3}$ 自由基 $\left[\mathrm{CH}_{4}+\mathrm{H} \rightarrow \mathrm{CH}_{3}+\mathrm{H}_{2}\right.$ (52.5\%)] 和 $\mathrm{CH}_{3}$ 自由基加成反应生成 $\mathrm{C}_{2} \mathrm{H}_{5}$ 自由基 $\left[\mathrm{CH}_{4}+\mathrm{CH}_{3} \rightarrow \mathrm{C}_{2} \mathrm{H}_{5}+\mathrm{H}_{2}(42.4 \%)\right]$ (图3a)。随后, 这些 $\mathrm{CH}_{3}$ 和 $\mathrm{C}_{2} \mathrm{H}_{5}$ 自由基会继续发生反应, 形成 $\mathrm{C}_{2} \mathrm{H}_{4} 、 \mathrm{C}_{2} \mathrm{H}_{6} 、 \mathrm{C}_{2} \mathrm{H}_{3} 、 \mathrm{C}_{3} \mathrm{H}_{3} 、 \mathrm{C}_{3} \mathrm{H}_{6} 、 \mathrm{C}_{6} \mathrm{H}_{5}$ 和 $\mathrm{C}_{6} \mathrm{H}_{6}$ 等 分子量更大的活性碳物种和碳团簇 62 。温度是气相 中的甲烷裂解反应的关键影响因素。一般来说, 低
温下气相中的组分多以分子形式存在; 当体系温 度大于 $700{ }^{\circ} \mathrm{C}$ 时, 气相中产生了大量 $\mathrm{CH}_{3}$ 自由基; 随 着体系温度的进一步升高, $\mathrm{CH}_{3}$ 自由基继续脱氢裂 解, 形成 $\mathrm{CH}_{2}$ 或 $\mathrm{CH}$ 自由基。

从热力学平衡的角度, 也可以对不同 CVD反 应条件下气相中的物种组成进行定量分析。如图 $3 \mathrm{~b}$ 所示, 侯建国课题组 ${ }^{63}$ 模拟了不同温度下CVD体 系内的物种组成, 发现气相中能够稳定存在的物 种多达 15 种，包括 $\mathrm{H} 、 \mathrm{H}_{2} 、 \mathrm{C} 、 \mathrm{CH} 、 \mathrm{CH}_{2} 、 \mathrm{CH}_{3} 、 \mathrm{CH}_{4} 、$ $\mathrm{C}_{2} 、 \mathrm{C}_{2} \mathrm{H} 、 \mathrm{C}_{2} \mathrm{H}_{2} 、 \mathrm{C}_{2} \mathrm{H}_{4} 、 \mathrm{C}_{2} \mathrm{H}_{6}$ 等。无独有偶, Ian A. Kinloch课题组 ${ }^{64}$ 的模拟结果同样证明, 在常规的 石墨烯生长条件下 $\left(1 \mathrm{mbar}, 1000^{\circ} \mathrm{C}\right)(1 \mathrm{mbar}=100$ $\mathrm{Pa})$, 气相物种组成复杂, 除 $\mathrm{CH}_{x} 、 \mathrm{C}_{2} \mathrm{H}_{y} 、 \mathrm{C}_{3} \mathrm{H}_{z}$ 等, 还存在分子量较大的活性碳物种, 如 $\mathrm{C}_{4} \mathrm{H}_{2} 、 \mathrm{C}_{6} \mathrm{H}_{6}$ 、 $\mathrm{C}_{7} \mathrm{H}_{8} 、 \mathrm{C}_{8} \mathrm{H}_{8} 、 \mathrm{C}_{10} \mathrm{H}_{8}$ 等。温度和压强等反应参数会 影响到气相物种的组成和最终的反应产物(图3c)。 如图3d, e所示, 相同的反应温度下, 当CVD体系 的压力 $P_{\mathrm{A}}$ 较大时 $(100 \mathrm{mbar})$, 气相中 $\mathrm{C}_{10} \mathrm{H}_{8}$ 和 $\mathrm{C}_{6} \mathrm{H}_{6}$ 等分子量较大的活性碳物种的含量相对较多, 即 使温度升高到 $1200{ }^{\circ} \mathrm{C}$, 它们的含量仍没有明显降 低; 而当体系压强较低时, 分子量较大的活性碳物 (a)

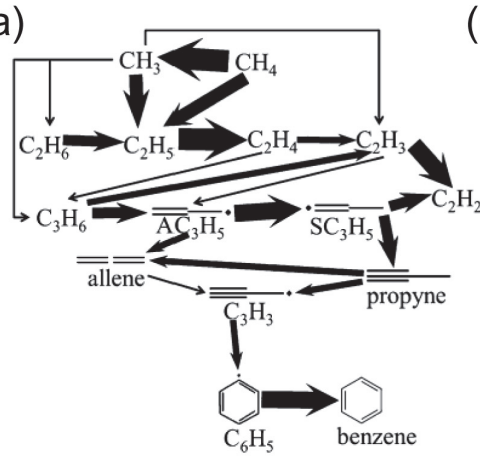

(c)

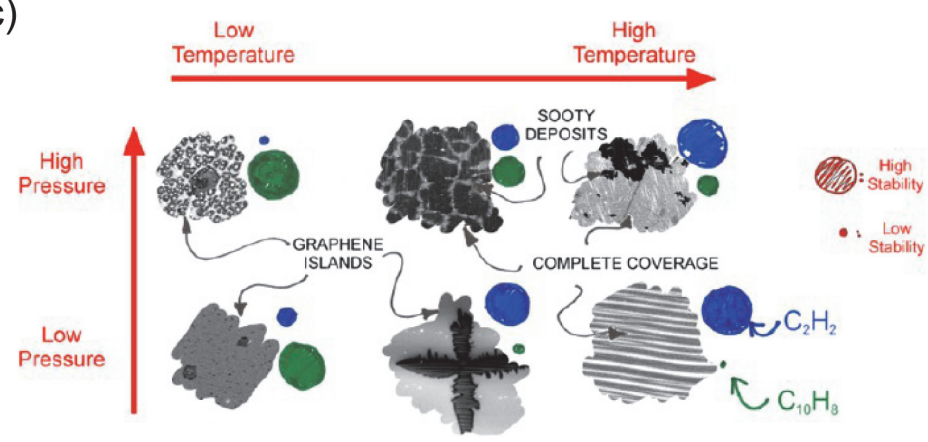

(b)

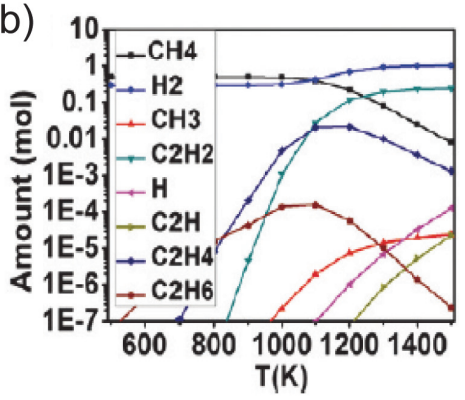

(d)

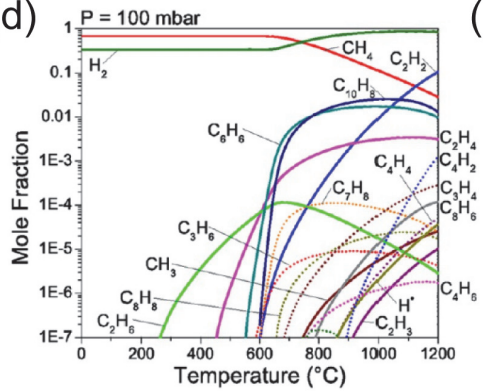

(e)

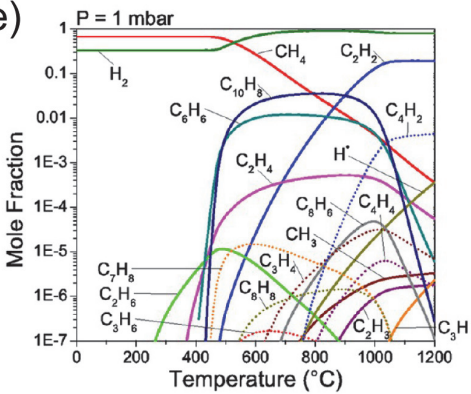

图 3 石墨烯高温 CVD 生长过程中的气相反应 ${ }^{62-64}$

Fig. 3 Gas-phase reactions during the high-temperature CVD growth of graphene ${ }^{62-64}$.

(a) High-temperature reaction path of methane in the gas phase of CVD system, where the thickness of the arrow indicates the reaction probability;

Adapted from Springer Nature publisher. (b) The concentrations of 15 species in the gas phase as a function of temperature; Adapted with permission from Ref. 63. Copyright 2012, American Chemical Society. (c) Illustration of the impact of pressure and temperature on the quality of graphene films deposited on the substrate; (d-e) Temperature-dependent composition variation of gas mixture under pressures of 100 mbar (d) and 1 mbar (e), respectively. 
种的含量随着温度的升高先增加后减少。一般来 说, 气相中分子量大的碳团簇的含量较多时, 石墨 烯的结晶质量往往不理想。这与实验上观察到的低 压高温的反应条件有利于生长高品质石墨烯的结 论相吻合 64 。

需要指出的是, 在引入金属祄底后, 气相反应 的平衡态被打破, 衬底消耗了大量活性碳物种用 于石墨烯的生长, 气相物种的类型和含量都有明 显变化。这也是金属祄底和绝缘祄底表面石墨烯 生长行为差异性的原因之一。例如, 侯建国课题 组 ${ }^{63}$ 通过理论计算发现, 当考虑铜祄底对气相中 $\mathrm{CH}_{3}$ 物种的吸附作用时, 吸附在祄底表面的活性碳 物种的含量会显著影响气相中的物种组成, 导致 气相物种的种类和含量明显减少。此外, 研究人员 通过理论计算还发现, 在没有金属催化时, 碳源裂 解只能通过热裂解进行, $\mathrm{C}_{2} \mathrm{H}_{5}$ 物种的含量很高; 而 当引入金属催化剂后, $\mathrm{C}_{2} \mathrm{H}_{5}$ 物种会发生催化裂解 脱氢反应, 同时伴随着 $\mathrm{C}_{2} \mathrm{H}$ 物种浓度的迅速增加 ${ }^{65}$ 。

除通过理论计算研究CVD体系中碳源裂解和 碳团簇形成等气相反应外, 研究人员也借助质谱 和紫外可见吸收光谱等手段在实验上探测到了 $\mathrm{CVD}$ 体系中的气相产物。例如, 通过在CVD体系 下游原位连接热重质谱仪, 李雪松课题组 37 发现气 相物种中除了分子量小于 20 的甲烷裂解产物, 还 有分子量大于 40 的产物, 这说明气相反应中碳源 裂解会产生活性碳物种, 并能进一步反应生成分 子量更大的碳团簇。此外, 研究人员通过紫外可见 吸收光谱对 CVD高温生长石墨烯过程中的气相产 物进行原位表征, 同样证实了边界层内活性碳物 种的存在和碳源裂解反应的发生 ${ }^{66}$ 。

除温度和压强外, 碳源类型也会影响 CVD体 系内的气相物种组成。这是因为, 不同碳源的裂解 势垒不同, 相同反应条件下的裂解产物也有明显 差异。例如, 甲烷裂解时会断裂 $\mathrm{C}-\mathrm{H}$ 键, 裂解产 物以 $\mathrm{CH}_{3}$ 自由基为主, 而乙炔则会优先断裂 $\mathrm{C} \equiv \mathrm{C}$ 键, 裂解产物以 $\mathrm{C}_{2} \mathrm{H}_{2}$ 自由基为主 ${ }^{67}$; 乙醇中 $\mathrm{C}-\mathrm{O}$ 键 的键能低于乙烷中 $\mathrm{C}-\mathrm{H}$ 键的键能, $\mathrm{C}-\mathrm{O}$ 键会优先 断裂, 大大降低碳源的裂解势垒 68 。

除碳源类型外, 氢气裂解产生的活性氢也能 促进碳氢键活化和脱氢反应的进行 ${ }^{50}$, 并影响碳氢 物种的类型和比例。一般认为, 氢气分压越大, $\mathrm{C}_{2} \mathrm{H}_{2}$ 物种的含量越多, 石墨烯的质量越高 69 。此外, 在 石墨烯生长过程中引入氧 $7^{70}$ 、氟 ${ }^{71}$ 等元素或金属催 化剂等 ${ }^{72}$ 也能够有效降低碳源的裂解势垒, 促进其 充分裂解, 进而显著改变气相反应, 并最终影响石 墨烯的生长行为和薄膜质量。
此外, 由于气相传质过程, CVD体系内的气相 反应和气相物种的组成还具有位置依赖性。研究 人员通过流体动力学计算发现, 从上游到下游, 气 相中 $\mathrm{CH}_{4}$ 的浓度逐渐降低, 而 $\mathrm{CH}_{3}$ 和 $\mathrm{C}_{2} \mathrm{H}$ 浓度不断 提高 65 。也就是说, 下游的碳源往往比上游裂解更 为充分, 且活性碳物种含量更高, 这就导致下游的 石墨烯薄膜成核密度更高、生长速度更快、覆盖度 更高且层数更厚。

\section{3 气相调控对石墨烯生长的影响}

\section{1 结晶性}

材料的结构决定其性质。石墨烯中的碳原子 以 $s p^{2}$ 杂化类型形成的单原子层厚度的六方蜂窝状 结构是其诸多优异性质的基础。石墨烯晶格中一 旦出现点缺陷、线缺陷等不完美结构, 它们就有可 能成为载流子、声子等的散射中心, 对石墨烯的性 质带来不利影响 73,74 。因此, 降低石墨烯的缺陷密 度, 提高其结晶性是保证石墨烯优异性能发挥的 前提和基础。

一般来说，铜、镍等金属祄底可以在石墨烯高 温生长过程中, 对点缺陷等不完美结构进行动态 修复 75,76 。因此, 在合适的反应温度下, 金属祄底 表面生长的本征石墨烯薄膜一般具有较好的结晶 性, Raman光谱检测结果中看不到明显的缺陷峰。 早期研究结果表明, 气相中金属催化剂含量的增 加也有助于进一步提高石墨烯薄膜的结晶性。例 如, 研究人员分别将铜䇴放入单端开口的石英套 管的两端进行石墨烯的生长, 由于靠近套管封口 位置处气相中的铜蒸气含量较高, 碳源裂解更充 分, 此处生长的石墨烯薄膜质量优于靠近套管开 口位置处的石墨烯77。

由于缺少促进碳源裂解和缺陷修复的金属催 化剂, 在绝缘祄底表面制备的石墨烯的结晶质量 通常并不理想(图4a) ${ }^{78}$ 。为解决这一问题, 可以通 过向CVD体系中引入金属蒸气作为气相催化剂, 促 进碳源裂解和绝缘祄底表面石墨烯的缺陷修复, 从而提高石墨烯的结晶性 ${ }^{31}$ 。如图 $4 \mathrm{~b}$ 所示, 沿载气 流动方向, 在绝缘祄底上游放置一块铜䈃, 即可显 著提高绝缘祄底上石墨烯薄膜的结晶质量。此时, 石墨烯样品Raman光谱的 $\mathrm{G}$ 峰强度与绝缘衬底和 铜箔之间的距离直接相关(图4c)。距离铜箔太近的 位置, 未检测到石墨烯的信号; 而距离铜䈃太远的 位置, 石墨烯的结晶质量也有所下降。这是因为高 温低压条件下, 放置在上游的铜箔不断挥发产生 铜蒸气, 铜蒸气在气相传质过程中不断向下游迁 移, 导致CVD体系内不同位置的铜蒸气含量不同。 
(a)

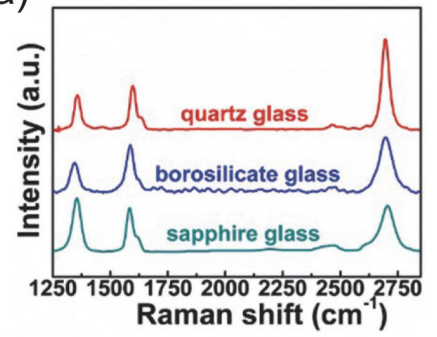

(c)

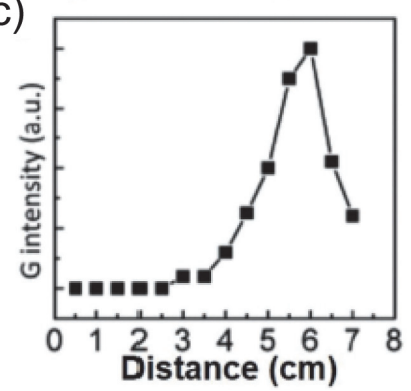

(b)

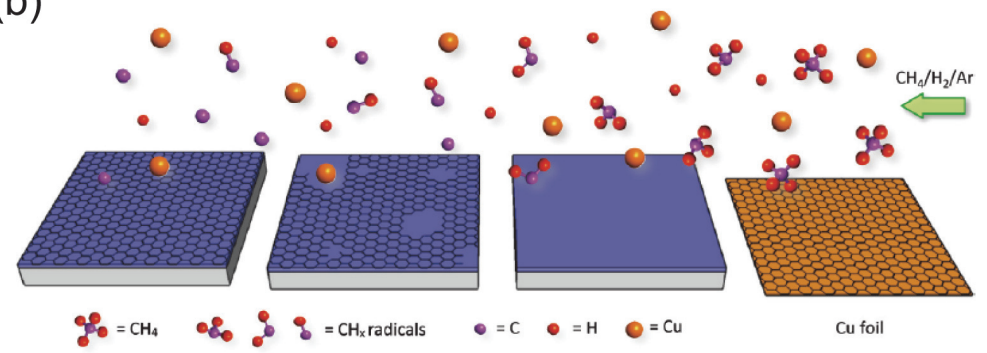

(d)

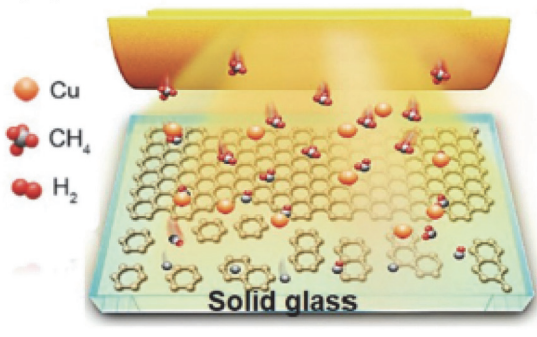

(e)

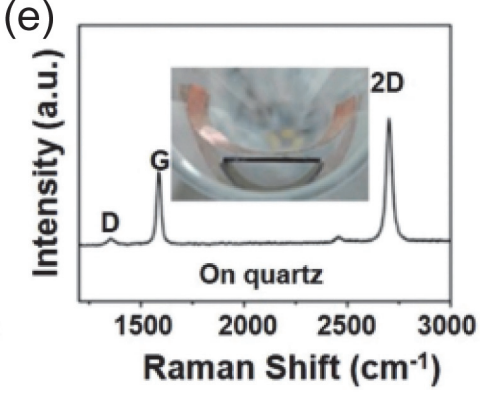

图 4 气相反应对石墨烯结晶性的影响 31,78,79

Fig. 4 Influence of gas-phase reactions on crystallinity of graphene ${ }^{31,78,79}$.

(a) Typical Raman spectra of graphene directly grown on quartz glass (red), borosilicate glass (blue) and sapphire glass (green) substrates; Adapted with

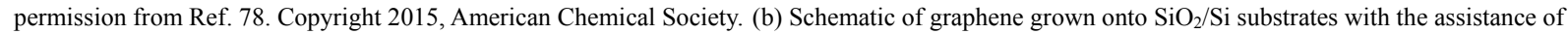
$\mathrm{Cu}$ vapor by placing the $\mathrm{Cu}$ foil in the upstream of the $\mathrm{SiO}_{2} / \mathrm{Si}$ substrates; (c) The Raman $\mathrm{G}$ peak intensity versus the distance between $\mathrm{Cu}$ foil and $\mathrm{SiO}_{2}$ substrates; Adapted with permission from Ref. 31. Copyright 2015, American Chemical Society. (d) Illustration of the graphene grown on solid glass with $\mathrm{Cu}$ foil placed on the top of the substrate; (e) Representative Raman spectrum of graphene grown on solid glass with the assistance of $\mathrm{Cu}$ vapor in the gas phase. Inset: the photograph of $\mathrm{Cu}$ foil-solid glass stacked structure for the growth of high-quality graphene.

Adapted with permission from Ref. 79. Copyright 2019, Wiley-VCH.

研究人员调整了引入金属蒸气的方式, 将放置在 上游的铜箔置于绝缘祄底的上方, 解决了铜箔挥 发出的铜蒸气在绝缘衬底表面分布不均匀的问 题, 在提高石墨烯薄膜结晶质量的同时, 也提高了 其生长的均匀性 $($ 图 $4 \mathrm{~d}, \mathrm{e}){ }^{79}$ 。除铜蒸气外, 向 CVD 体系内额外引入镍蒸气、镓蒸气或含氧蒸气等也 被证明可以显著提高绝缘祄底表面石墨烯薄膜的 结晶质量 $42,59,72$ 。

\section{2 洁净度}

高温 CVD体系中, 石墨烯的生长往往伴随着 大量气相副反应的发生, 导致石墨烯薄膜表面沉 积了大量的无定形碳, 造成石墨烯薄膜的 “本征污 染” 现象 ${ }^{80}$, 并对石墨烯材料的光、热、力、电等 性能带来不利影响 $32,55,81-84$ 。

为解决石墨烯的 “本征污染” 问题, 北京大学 刘忠范-彭海琳课题组提出了 “气相助催化” 的方 法, 通过向气相中持续不断地供给铜蒸气, 有效提 高了碳源在气相中的催化裂解程度, 减少了气相 中较大分子量的碳团簇的形成, 进而抑制了无定 形碳污染物的生成, 成功地制备出了超洁净石墨 烯薄膜。
他们尝试了两种策略, 其一是引入比表面积 大的泡沫铜, 其二是使用含铜碳源醋酸铜。泡沫铜 比表面积大, 在高温、低压CVD体系中可挥发出大 量铜蒸气(图5a), 保证气相中充足的铜蒸气供给 32 。 此外, 泡沫铜具有较强的吸附能力, 能吸附气相边 界层中的副反应产物, 进一步减少石墨烯表面可 能出现的污染物的含量。实验结果表明, 当引入泡 沫铜后, 石墨烯薄膜表面的污染物含量显著降低, 洁净度从不足 $50 \%$ 提高到了 $99 \%$ (图 5b, c)。图 $5 \mathrm{~d}$ 的 理论计算结果定量比较了气相中甲烷催化裂解和 热裂解的势垒差异, 进一步证实了气相金属催化 剂的存在对降低碳源脱氢势垒, 促进其充分裂解 的重要性。如图 $5 \mathrm{e}$ 所示, 含铜碳源醋酸铜能够在供 给活性碳物种的同时, 保证气相中铜蒸气的持续 稳定供给 55 。与使用甲烷相比, 使用醋酸铜作为碳 源能够有效抑制气相副反应的发生, 提高 CVD石 墨烯薄膜的表面洁净度。使用分子篮分别收集了 使用甲烷和醋酸铜生长石墨烯时的气相组分并进 行拉曼光谱表征可知(图5f): 使用甲烷生长石墨烯 时, 分子篮收集到的物种有明显的D峰, 说明气相 中存在大量结晶性较差的无定形碳; 而使用醋酸 
(a)

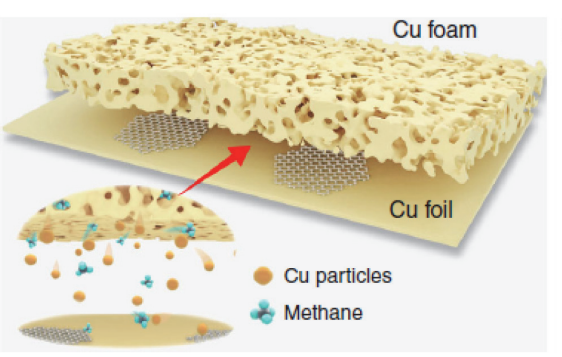

(b)

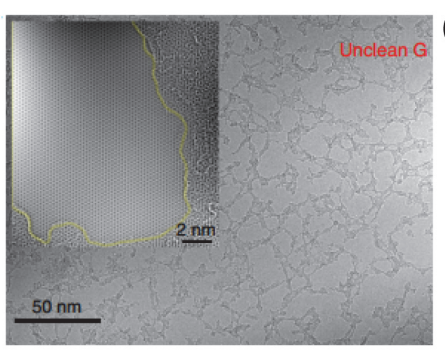

(c)

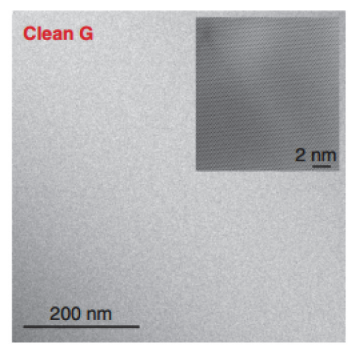

(e)

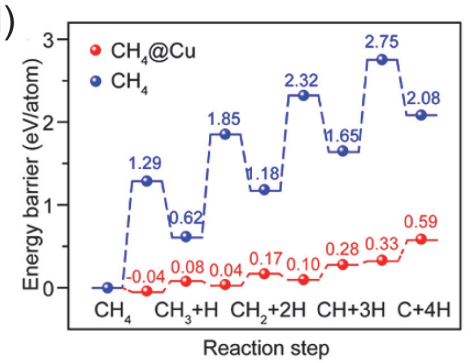

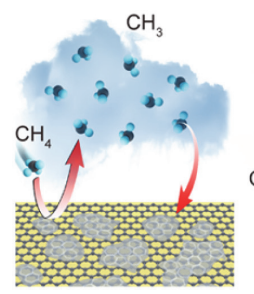

(f)

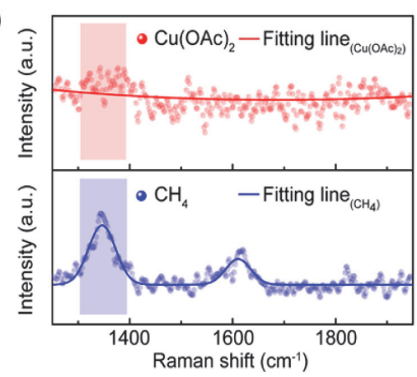

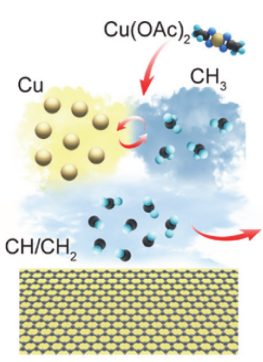

(g)

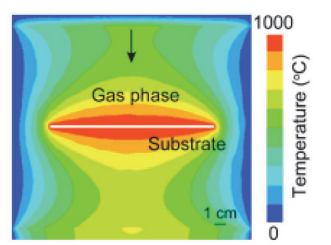

(h)

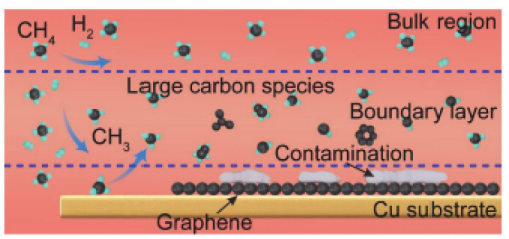

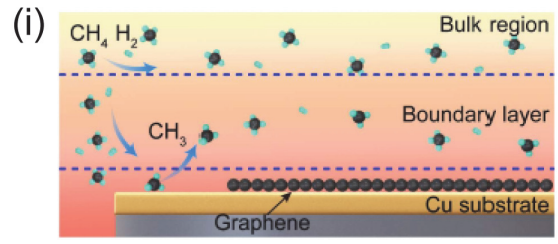

图 5 气相反应对石墨烯洁净度的影响 $32,55,83$

Fig. 5 Influence of gas-phase reactions on intrinsic cleanness of graphene ${ }^{32,55,83}$.

(a) Illustration of the superclean growth of graphene with the assistance of $\mathrm{Cu}$ foam; (b, c) Typical TEM image of unclean (b) and superclean (c) graphene. Inset: HRTEM image of the unclean (b) and superclean (c) graphene with atomic resolution; Adapted with permission from Ref. 32. Copyright 2019, Springer Nature. (d) Energy barriers of the decomposition of $\mathrm{CH}_{4}$ in the gas phase without (blue) and with (red) the participation of $\mathrm{Cu}$ vapor; (e) Schematic diagram of the growth of graphene with $\mathrm{CH}_{4}$ (left) and $\mathrm{Cu}(\mathrm{OAc})_{2}$ (right) as carbon source; (f) Representative Raman spectra of the carbon species formed during the growth of graphene using $\mathrm{Cu}(\mathrm{OAc})_{2}$ (red) and $\mathrm{CH}_{4}$ (blue). Adapted with permission from Ref. 55. Copyright 2019, American Chemical Society.

(g) The simulated temperature distribution in cold-wall CVD (CW-CVD) system. (h, i) Schematic of reaction in the boundary layer in the hot-wall CVD (h) and CW-CVD (i) system. Adapted with permission from Ref. 83. Copyright 2020, Wiley-VCH.

铜生长石墨烯时, 收集的物种却几乎没有 $\mathrm{D}$ 峰, 说 明气相中几乎没有无定形碳, 即充足的铜蒸气能 够有效抑制气相中副产物的形成。

除金属催化剂外, 冷壁CVD体系中独特的温 场分布 (图 $5 \mathrm{~g}$ ) 也能抑制气相中大的碳团簇的形 成, 减少 $\mathrm{CVD}$ 生长的石墨烯薄膜表面污染物的含 量。在第二节的讨论中已经提到, 随着温度的升 高, 气相中分子量较大的活性碳物种的含量也会 有所增加。传统的热壁CVD通过对反应器壁加热 使整个体系处于高温状态, 气相温度与祄底温度 基本相同, 高温下气相中形成的大量活性碳氢物 种, 容易碰撞形成大的碳团簇, 导致石墨烯表面无 定形碳的生成(图5h)。与热壁 CVD不同, 冷壁 CVD 只需将衬底加热到石墨烯所需的生长温度, 而气 相可保持较低的温度, 在高温生长石墨烯的同时, 能够有效抑制气相副反应的发生(图5i)。基于此, 研 究人员利用冷壁CVD中独特的温场分布制备出了
超洁净石墨烯薄膜 83 , 为高品质石墨烯薄膜的工业 化生产提供了一种新选择。

绝缘祄底表面生长的石墨烯薄膜同样存在本 征污染的问题, 且无定形碳污染物往往含量更多。 对于绝缘祄底表面石墨烯薄膜的生长, 除了在气 相中引入金属蒸气外 ${ }^{32}$, 通入合适的氧化性气氛, 也能够刻蚀石墨烯表面的污染物, 获得高洁净度 的石墨烯薄膜。例如, 研究人员在高温生长石墨烯 的过程中, 向 CVD体系内通入微量的水蒸气对石 墨烯表面的无定形碳进行刻蚀, 并通过改变水蒸 气的含量, 实现了石墨烯薄膜洁净度的有效调控 85 。 在一定范围内, 随着水蒸气含量的增加 $(0-0.3 \%$, volume fraction), 石墨烯薄膜的洁净度不断增加。 此外, 选用含氧碳源乙醇等也能提高石墨烯薄膜 的表面洁净度 ${ }^{86}$ 。

\section{3 石墨烯层数}

石墨烯的层数对其性质和应用有显著影响 ${ }^{87}$ 。 
以石墨烯的电学性质为例, 单层石墨烯是零带隙 半金属材料 ${ }^{13}$; 而双层 $\mathrm{AB}$ 堆垛的石墨烯由于层间 $\pi$ 轨道的耦合, 在施加外电场后很容易打开带隙成 为半导体 ${ }^{79}$, 在光电子应用和未来的微处理器方面 有独特的应用优势。因此, 控制石墨烯的层数和大 面积层数均匀性对于实际应用具有重要意义 ${ }^{88}$ 。

如前所述, 气体进入CVD系统后, 在石英管内 沿其轴向流动, 碳源裂解产生的活性碳物种浓度 沿着气流方向不断增加, 且下游碳源物种裂解更 充分(图6a), 导致上下游石墨烯的层数出现差异且 下游更容易生成双层和少层石墨烯 63 。如图 $6 \mathrm{~b}$ 所 示, 研究人员对体系内不同位置生长的石墨烯进 行透光率测试, 发现样品存在明显的不均匀性, 上 游到下游石墨烯的透光率从 $97.2 \%$ 降低到 $78.5 \%$, 即从上游石墨烯以单层为主, 而下游石墨烯的的 厚度明显增加, 根据透光率折算, 相当于9-10层石 墨烯的厚度。而使用水平等间距放置的多片铜箔 (图6a), 由于不同位置处金属催化剂含量和碳源裂 解程度的改变, 气相中活性碳物种分布的均匀性 得到了有效提升, 大范围内石墨烯薄膜的层数也 更加均匀, 其透光率变化范围仅为 $0.8 \%(96.6 \%-$ 97.4\%), 即不同位置的石墨烯薄膜均以单层为主 (图6c)。当批量制备大尺寸石墨烯薄膜时, 随着 CVD体系尺寸的增大, 气相传质过程也会变得更 加复杂 ${ }^{89}$, 这进一步增加了大尺寸石墨烯薄膜层数 控制的难度。因此, 气体流动状态和流场分布状态 的调控对于大尺寸石墨烯薄膜的层数控制尤为关 键 $^{90}$ 。比如, 为了提高铜薄膜/蓝宝石祄底表面批量 制备的石墨烯晶圆的层数均匀性, 研究人员将 CVD体系的进气孔由传统的单气孔供气调整为多 气孔供气, 并结合生长祄底的间距优化, 显著改善 了气相中流场分布的均匀性(图6d) ${ }^{89}$ 。为实现了单 批次 30 片 4 inch $(1$ inch $=2.54 \mathrm{~cm})$ 石墨烯/石英晶圆 的均匀生产, 研究人员结合流场动力学模拟, 提出 (a)

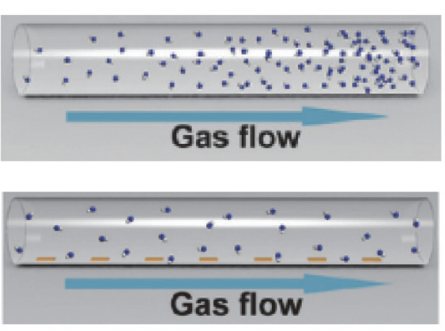

(b)

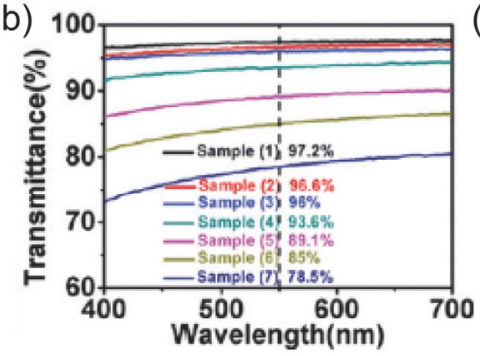

(d)

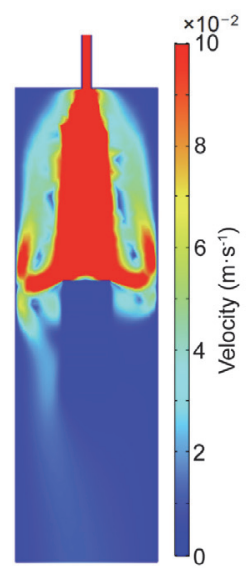

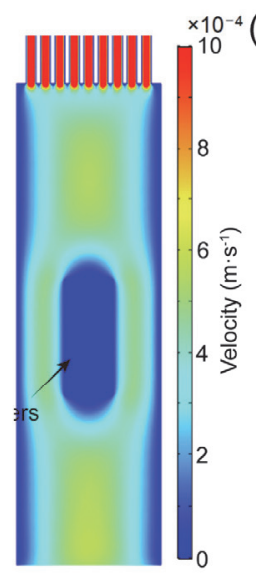

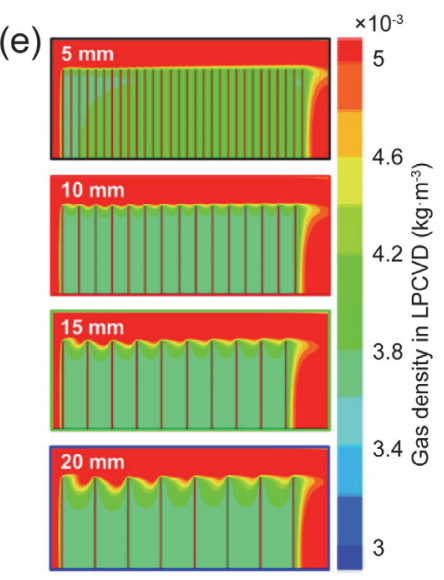

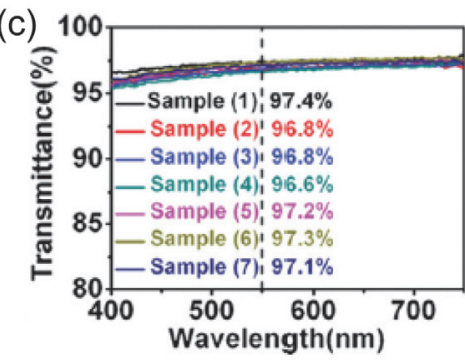

(f)

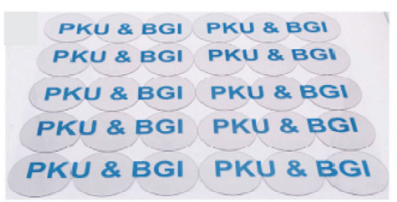

(g)

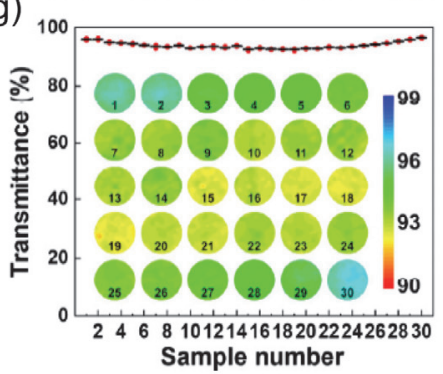

图 6 气相反应对石墨烯层数的影响 $63,89,91$

Fig. 6 Influence of gas-phase reactions on graphene layer ${ }^{63,89,91}$.

(a) Schematic diagram of the concentration distribution of the active carbon species when seven different positions independently (top) and seven separated $\mathrm{Cu}$ foils simultaneously (bottom) were placed along the tube; $(\mathrm{b}-\mathrm{c}) \mathrm{UV}$-vis spectra of the graphene films grown on seven different positions independently (b) and seven separated $\mathrm{Cu}$ foils simultaneously (c). Note that samples 1 to 7 are placed along the direction of the gas flow. Adapted with permission from Ref. 63. Copyright 2012, American Chemical Society. (d) Distribution of the gas flow velocity in the CVD system with a single gas nozzle (left) and multiple gas nozzles (right) across the chamber; Adapted from Elsevier publisher. (e) Simulation of the gas density distribution in LPCVD with various intervals of quartz wafers; (f) Photograph of 30 pieces of graphene/quartz wafers grown in one batch.; (g) Average transmittance of 30 pieces of graphene/quartz 
了新的祄底放置方案, 有效提高绝缘祄底上批量 制备石墨烯的流场均匀性(图6e) ${ }^{91}$ 。该方法制备的 石墨烯晶圆面内和批次内都具有良好的层数均匀 性, 且透光率均高于 $90 \%$ (图6f，g)。

\section{4 畴区尺寸}

在石墨烯薄膜生长过程中, 畴区取向不一致 的相邻畴区拼接时会产生晶界。晶界的存在会严 重影响石墨烯的电、热、力学等性能和化学稳定 性 ${ }^{92-94}$ 。因此, 增大石墨烯的畴区尺寸, 减少晶界 数量一直是高品质石墨烯生长的重要目标。

降低石墨烯的成核密度是增大石墨烯畴区尺 寸的关键 ${ }^{49,95}$ 。其中, 气相中碳源的供给方式对石 墨烯的成核密度影响很大。如图7a所示, 研究人员 采用局域供碳的方式, 使得 $\mathrm{Cu}_{85} \mathrm{Ni}_{15}$ 合金祄底上的 局域碳浓度过饱和, 而其他区域碳浓度几乎为零, 实现了石墨烯在单个位点的成核, 生长 $150 \mathrm{~min}$ 即 可得到畴区尺寸为 $1.5 \mathrm{inch}$ 的石墨烯单晶 ${ }^{34}$ 。而在 相同的生长条件下, 若采用全局供碳, 合金祄底上 会有较多的成核位点, 仅能得到毫米尺寸的多个 石墨烯单晶(图7b)。如图7c所示, 研究人员将局域 供碳与石墨烯动态生长相结合, 利用 “进化选择” 生长法, 在多晶铜镍合金祄底上成功实现了英尺 级别石墨烯畴区的制备 ${ }^{96}$ 。

使用绝缘衬底生长石墨烯时, 热裂解反应产 生的活性碳物种供应不足，会导致石墨烯的生长 受限, 畴区尺寸较小。为了促进碳源的充分裂解, 研究人员使用含有金属的二茂镍作为碳源，借助 气相中镍蒸气的催化裂解作用(图7d), 有效提高了 绝缘祄底上石墨烯的畴区尺寸和生长速度 ${ }^{72}$ 。由图 $7 \mathrm{e}$ 可知, 石墨烯畴区的最大尺寸可达 $20 \mu \mathrm{m}$, 显著 优于相同生长时间内使用苯甲酸和甲烷作为碳源 生长的石墨烯的畴区尺寸。

\section{5 生长速度}

新兴材料广泛应用的关键在于低成本批量化 制备。CVD法生长石墨烯通常需在较高温度进行, 生长时间越长, 能耗越大。因此, 石墨烯生长速度 的提升, 能够减少生长石墨烯所需的时间, 助力石 墨烯薄膜的低成本制备。 (a)

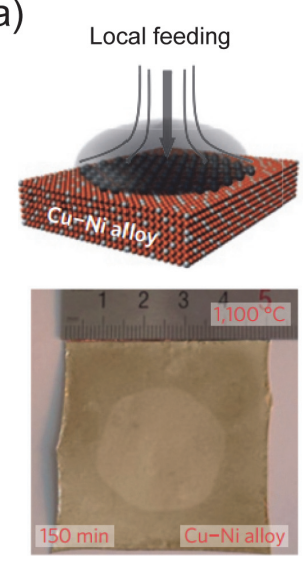

(b)
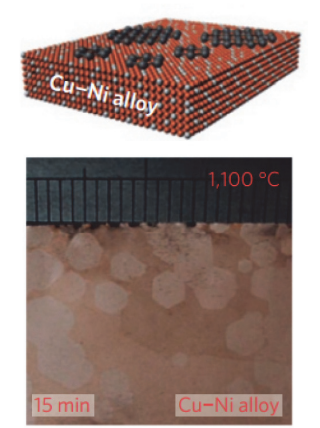

(c)
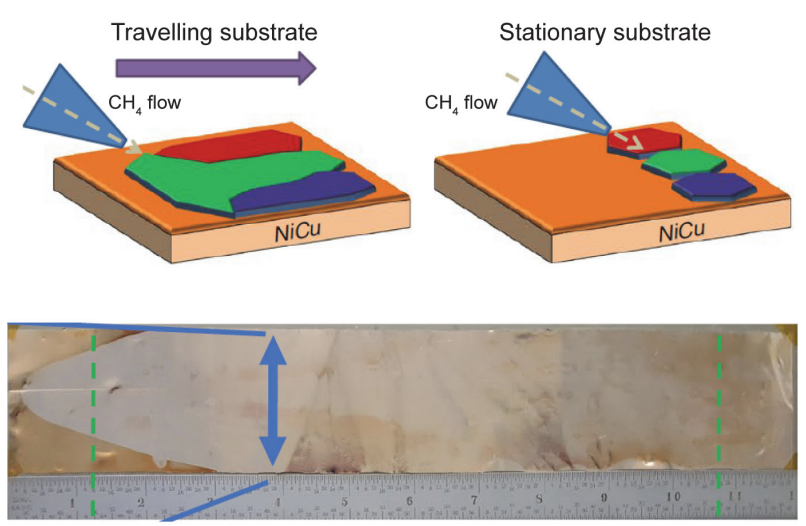

(e)

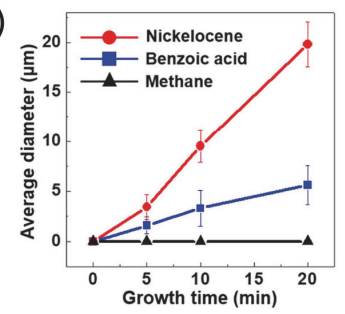

(d)

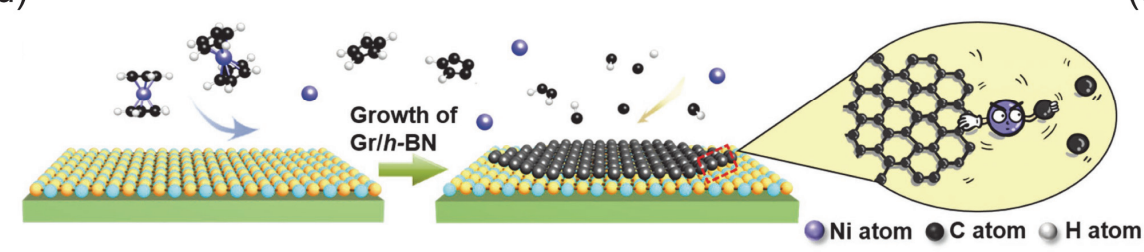

图 7 气相反应对石墨烯畴区尺寸的影响 34,72,96

Fig. 7 Influence of gas-phase reactions on domain size of graphene ${ }^{34,72,96}$.

(a) Schematic of single-crystal graphene growth on $\mathrm{Cu}_{85} \mathrm{Ni}_{15}$ alloy substrate by using the quartz with a tiny nozzle (top) and the typical photograph of graphene single crystal (bottom); (b) Schematic of graphene growth on $\mathrm{Cu}_{85} \mathrm{Ni}_{15}$ alloy substrate by introducing the carbon precursors homogeneously into the CVD system (top) and the corresponding photograph of graphene domains (bottom); Adapted from Springer Nature publisher. (c) Illustration of graphene growth on travelling (top, left) and stationary (top, right) substrate and the corresponding photograph of single-crystal graphene grown by evolutionary selection growth; Adapted from Springer Nature publisher. (d) Illustration of graphene growth on h-BN/Cu foils using nickelocene as the precursor; (e) Relationship between the domain size of graphene and growth time using nickelocene (red), benzoic acid (blue), and methane (black) as precursors, respectively. Adapted with permission from Ref. 72. Copyright 2017, Wiley-VCH. 
活性碳物种的供给是影响石墨烯生长速度的 关键因素。基于气相传质部分的讨论可知, 构建限 域空间可以将气体流动状态由粘滞流改为分子 流, 增加气相物种与祄底的碰撞几率, 进而提高石 墨烯的生长速度。基于该原理, 研究人员采用新颖 的铜馢叠层堆垛的方式(图8a), 实现了石墨烯的快 速生长 ${ }^{97}$ 。他们将垂直堆垛的铜箔间隙控制在 $10-$ $30 \mu \mathrm{m}$, 该距离远小于常规LPVCD体系中气体分子 的平均自由程, 保证了气体分子的运动状态为分 子流模式。限域空间的存在促使气体分子在堆垛 的铜箔祄底间来回碰撞, 分子间的碰撞频率显著 提高, 有效促进了碳源的裂解, 从而提升了祄底表 面的活性碳物种浓度。当使用甲烷作为碳源生长 石墨烯时, 仅生长 $10 \mathrm{~min}$ 即可获得 $3 \mathrm{~mm}$ 的石墨烯 畴区(图 $8 \mathrm{~b})$, 即石墨烯的最快生长速度可达 300 $\mu \mathrm{m} \cdot \mathrm{min}^{-1}$ 。

基于限域空间的设计, 若引入其他辅助因素, 石墨烯的生长速度可进一步提高。同样基于铜簿 垂直堆垛的限域空间设计, 将碳源从甲烷换为裂 解势垒更低的乙烷, 能够将石墨烯的生长速度进
一步提高到 $420 \mu \mathrm{m} \cdot \mathrm{min}^{-1}$, 实现亚厘米尺寸石墨烯 单晶的快速制备(图8c) ${ }^{98}$ 。此外, 在限域空间内, 引 入微量氧、氟等能够降低碳源裂解势垒, 从而更加 显著地提高石墨烯的生长速度。例如, 研究人员将 平整的氧化物基板置于铜䇴下方, 向 $\mathrm{CVD}$ 体系内 提供连续的氧供应, 实现了最高可达 $3.6 \mathrm{~mm} \cdot \mathrm{min}^{-1}$ 的生长速度 ${ }^{70}$; 将金属氟化物基板置于铜箔下方, 提供连续的氟供给, 实现了最高可达 $12 \mathrm{~mm} \cdot \mathrm{min}^{-1}$ 的生长速度 ${ }^{71}$ 。氟化物辅助石墨烯快速生长的策 略，对于绝缘衬底同样适用 99 。

除铜 “信封” 和铜䇴垂直堆垛的结构外, 大尺 寸铜祄底表面生长石墨烯时, 限域空间的构建往 往需要借助载具来对铜䇴辅助支撑, 以避免上下 层铜箔之间的黏连。此时, 除水平放置外, 衬底也 可以垂直放置 100 。例如, 研究人员基于特殊的载具 设计, 实现了多片生长祄底相互平行的垂直放置, 通过优化载具间的距离, 对石墨烯的生长速度实 现了有效调控 101 。

在绝缘祄底表面生长石墨烯时, 构建限域空 间同样能够提高石墨烯的生长速度。研究人员把

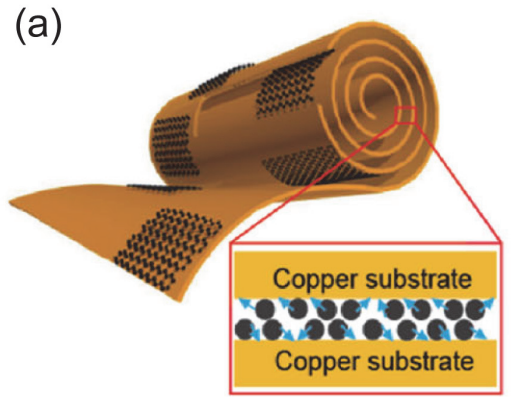

(b)

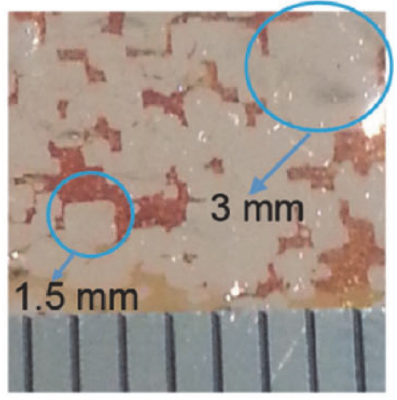

(c)

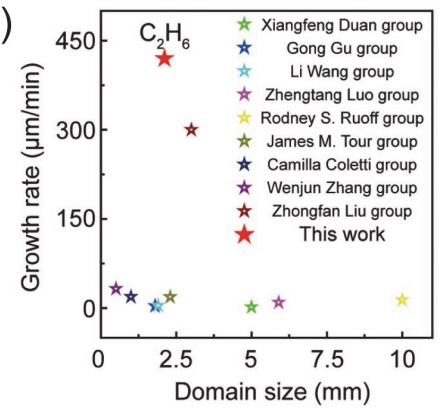

(d)

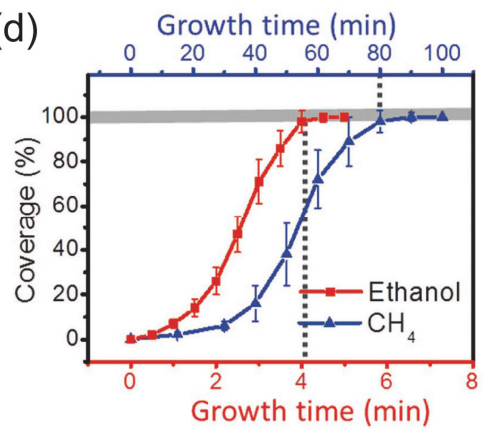

(e)

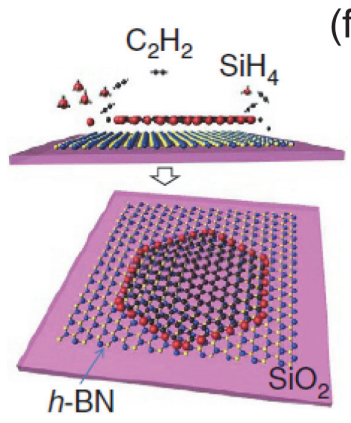

(f)

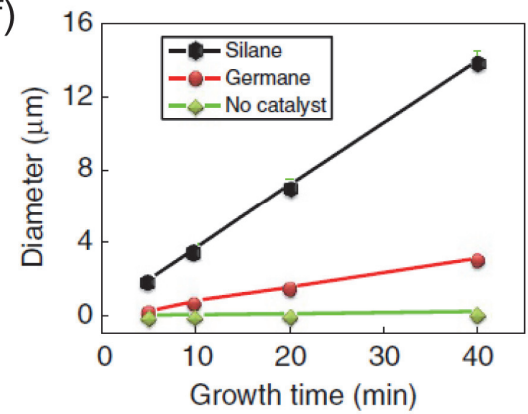

图 8 气相反应对石墨烯生长速度的影响 $35,86,97,98$

Fig. 8 Influence of gas-phase reactions on growth rate of graphene ${ }^{35,86,97,98}$.

(a) Schematic of the fast growth of single-crystal graphene on roll of $\mathrm{Cu}$ foils under free molecular flow; (b) Photograph of the as-produced graphene domains grown on the inner surface of the $\mathrm{Cu}$ foils; Adapted with permission from Ref. 97. Copyright 2016, Wiley-VCH. (c) Growth rate of single-crystal graphene using ethane comparing with other previously reported works; Adapted with permission from Ref. 98. Copyright 2018, Wiley-VCH. (d) Coverage of graphene grown on glass as a function of the growth time using ethanol (red) and methane (blue) as carbon sources; Adapted with permission from Ref. 86. Copyright 2017, Wiley-VCH. (e) Illustration of the growth of graphene on h-BN with the gaseous catalyst-assisted method; (f) The growth duration versus the growth time of graphene with silane (black), germane (red), and no catalyst (green). Adapted with permission from Ref. 35. Copyright 2015, Springer Nature. 
一片毛玻璃置于绝缘祄底上方, 在两者之间形成 2-4 $\mu \mathrm{m}$ 高的狭缝作为 “限域反应室”, 在石墨烯生 长过程中, 毛玻璃粗糙的表面对反应气流造成扰 动, 有效地增加了碳源分子之间的碰撞频率, 提高 了活性碳物种的局域浓度。该方法能够在 $75 \mathrm{~min}$ 内 获得满覆盖的石墨烯薄膜, 其生长速度远高于绝 缘社底上石墨烯的常规生长速度 ${ }^{102}$ 。

碳源类型对绝缘祄底上石墨烯薄膜的生长速 度同样有着显著影响。在相同生长温度下, 裂解势 垒较低的碳源会产生更多的活性碳物种, 从而提 高石墨烯的生长速度。例如, 研究人员利用乙醇作 为碳源, 进一步提升了石墨烯的生长速率, 在 $4 \mathrm{~min}$ 内即可制备出 $60 \mathrm{~cm}$ 长度的满覆盖的石墨烯薄膜 (图8d) ${ }^{86}$ 。

此外, 选用合适的气相催化剂也能提高石墨 烯的生长速度 ${ }^{103}$ 。例如, 利用硅烷 ${ }^{35}$ 作为气相催化 剂能实现石墨烯在六方氮化嗍上的快速生长(图 $8 \mathrm{e}, \mathrm{f})$, 石墨烯的畴区可以在 $15 \mathrm{~min}$ 内长大至 15 $\mu \mathrm{m}$ 。这是因为引入的硅原子能够降低活性碳物种 拼接到石墨烯边缘的势垒。与未引入硅烷时石墨 烯的生长速率相比, 该方法中石墨烯的生长速率 提高了两个数量级。选用含有金属的碳源, 如二茂 镍 ${ }^{72}$ 、醋酸铜 5 等同样能达到上述效果。

\section{4 总结}

本文综述了气相反应对 $\mathrm{CVD}$ 生长石墨烯的影 响, 系统总结了通过气相调控法制备高品质石墨 烯的代表性工作。我们从石墨烯 CVD生长的基元 步骤出发, 介绍了相互制约的气相反应和祄底表 面反应对石墨烯生长行为的影响, 从气相传质过 程和气相反应两方面入手详细讨论了气相调控对 石墨烯生长的重要性和影响规律。随后, 我们列举 了近年来通过调控气相反应制备高品质石墨烯薄 膜的相关工作，阐述了气相传质过程和气相反应 的改变对石墨烯的结晶性、洁净度、畴区尺寸、层 数可控性和生长速度等的影响, 并对相关调控策 略的基本原理、关键参数和典型结果进行了介绍。

总体来说，目前调控气相反应制备高品质石 墨烯薄膜的策略可以分为以下几类: (1)增加气相 中金属催化剂含量, 促进碳源裂解, 包括使用含有 金属元素的碳源、金属箔材、金属泡沫或液态金属 等; (2)选择更易裂解的碳源, 例如乙烷、乙炔、乙 醇等; (3)引入含氧物种, 可以是氧气、水、二氧化 碳等小分子, 氧化铝、氧化硅等无机物或乙醇等含 氧碳源; (4)构筑限域空间, 使气体流动状态由粘 滞流变为分子流, 以增加气相物种与祄底的碰撞
频率, 具体可通过构筑铜 “信封”、铜箔垂直堆垛 结构、绝缘祄底垂直堆垛结构、铜䈃/绝缘祄底堆 垛结构等方式来实现, 在石墨烯薄膜批量制备过 程中巧妙的载具设计也能实现上述目的。

值得一提的是, 利用气相调控制备高品质石 墨烯薄膜的策略对于金属和绝缘衬底上石墨烯的 生长具有较好的普适性, 能够相互借鉴和推广。例 如, 不论在金属祄底还是绝缘祄底上, 构建限域空 间都能提高碳源分子的碰撞频率, 促进碳源的裂 解, 进而提高石墨烯的生长速率。与此同时, 气相 调控策略对于石墨烯的生长往往具有多方面的增 益效果。例如, 引入气相催化剂, 能够降低碳源的 脱氢势垒, 提高碳源的裂解程度和裂解速度, 进而 提高石墨烯薄膜的生长速度、畴区尺寸和表面洁 净度; 通过改变祄底的放置方式来调整气相传质 过程和流场均匀性, 可以同时改变石墨烯的畴区 尺寸、生长速度和层数均匀性等。

与实验室水平制备的小片的高品质石墨烯薄 膜相比，批量制备的大尺寸石墨烯薄膜的质量仍 有较大的提升空间。可以预见的是, 气相反应的调 控将在石墨烯薄膜的可控批量制备方面发挥着至 关重要的作用。如前所述, 气相反应和传质过程对 CVD体系的反应参数非常敏感: 温度、压强、体系 的特征长度、碳源类型、金属催化剂含量和祄底摆 放方式及位置等都会影响气相中的物种组成和分 布。一般来说, 气体的流动状态决定了气相物种与 衬底的碰撞频率, 对碳源裂解程度和速率影响很 大, 进而会影响到石墨烯的成核密度和生长速率; 而流场的均匀性则会影响活性碳物种的分布, 进 行影响石墨烯的层数均匀性。想要实现更高品质 的石墨烯薄膜的批量制备还需要我们对上述过程 继续开展更加深入系统的研究。

大尺寸石墨烯薄膜的批量制备，目前还面临 着一些挑战, 例如: 高品质石墨烯的低成本批量制 备需要专门的批量制备装备、生长工艺和气相传 质过程的优化设计; 金属铜祄底表面大面积、高均 匀性且层数可控的石墨烯薄膜的制备需要进一步 提升流场、热场均匀性和生长祄底的均匀性; 大尺 寸单晶超洁净石墨烯薄膜的可控制备需要对石墨 烯的生长过程, 尤其是气相反应的影响机制有更 加清晰的了解; 绝缘衬底表面生长的石墨烯薄膜 的结晶质量、畴区尺寸、层数控制和生长速度等都 还有很大的提升空间; 为实现高品质石墨烯薄膜 的规模化生长和应用, CVD石墨烯薄膜批量制备 的成本还需要进一步降低等。

我们相信通过系统梳理气相反应对 CVD生 长石墨烯的影响, 能够推动对这些问题的研究和 
解决, 为高品质石墨烯薄膜的可控制备提供新的 思路和启发, 并加快高品质石墨烯薄膜的产业化 进程。

\section{References}

(1) Du, X.; Skachko, I.; Barker, A.; Andrei, E. Y. Nat. Nanotechnol. 2008, 3, 491. doi: 10.1038/nnano.2008.199

(2) Balandin, A. A.; Ghosh, S.; Bao, W. Z.; Calizo, I.; Teweldebrhan, D.; Miao, F.; Lau, C. N. Nano Lett. 2008, 8, 902. doi: 10.1021/n10731872

(3) Nair, R. R.; Blake, P.; Grigorenko, A. N.; Novoselov, K. S.; Booth, T. J.; Stauber, T.; Peres, N. M. R.; Geim, A. K. Science 2008, 320, 1308 doi: $10.1126 /$ science. 1156965

(4) Lee, C.; Wei, X. D.; Kysar, J. W.; Hone, J. Science 2008, 321, 385 doi: $10.1126 /$ science. 1157996

(5) Stoller, M. D.; Park, S.; Zhu, Y.; An, J.; Ruoff, R. S. Nano Lett. 2008, 8, 3498. doi: $10.1021 / \mathrm{nl} 802558 \mathrm{y}$

(6) Lozada-Hidalgo, M.; Zhang, S.; Hu, S.; Esfandiar, A.; Grigorieva, I. V.; Geim, A. K. Nat. Commun. 2017, 8, 15215. doi: 10.1038/ncomms 15215

(7) Kou, L.; Huang, T. Q.; Zheng, B. N.; Han, Y.; Zhao, X. L.; Gopalsamy, K.; Sun, H. Y.; Gao, C. Nat. Commun. 2014, 5, 3754. doi: $10.1038 /$ ncomms 4754

(8) Liu, C.-H.; Chang, Y.-C.; Norris, T. B.; Zhong, Z. H. Nat. Nanotechnol. 2014, 9, 273. doi: 10.1038/nnano.2014.31

(9) Tran Thanh, T.; Nine, M. J.; Krebsz, M.; Pasinszki, T.; Coghlan, C. J.; Tran, D. N. H.; Losic, D. Adv. Funct. Mater. 2017, 27, 1702891. doi: 10.1002/adfm.201702891

(10) Han, N.; Cuong, T. V.; Han, M.; Ryu, B. D.; Chandramohan, S.; Park, J. B.; Kang, J. H.; Park, Y. J.; Ko, K. B.; Kim, H. Y.; et al. Nat. Commun. 2013, 4, 1452. doi: 10.1038/ncomms 2448

(11) Yan, Z.; Liu, G. X.; Khan, J. M.; Balandin, A. A. Nat. Commun. 2012, 3, 827. doi: $10.1038 /$ ncomms 1828

(12) Bonaccorso, F.; Colombo, L.; Yu, G. H.; Stoller, M.; Tozzini, V.; Ferrari, A. C.; Ruoff, R. S.; Pellegrini, V. Science 2015, 347, 1246501. doi: 10.1126/science. 1246501

(13) Novoselov, K. S.; Geim, A. K.; Morozov, S. V.; Jiang, D.; Zhang, Y.; Dubonos, S. V.; Grigorieva, I. V.; Firsov, A. A. Science 2004, 306, 666. doi: $10.1126 /$ science. 1102896

(14) Lu, X. K.; Yu, M. F.; Huang, H.; Ruoff, R. S. Nanotechnology 1999, 10, 269. doi: $10.1088 / 0957-4484 / 10 / 3 / 308$

(15) Blake, P.; Brimicombe, P. D.; Nair, R. R.; Booth, T. J.; Jiang, D.; Schedin, F.; Ponomarenko, L. A.; Morozov, S. V.; Gleeson, H. F.; Hill, E. W.; et al. Nano Lett. 2008, 8, 1704. doi: 10.1021/n1080649i

(16) Berger, C.; Song, Z. M.; Li, X. B.; Wu, X. S.; Brown, N.; Naud, C.; Mayou, D.; Li, T. B.; Hass, J.; Marchenkov, A. N.; et al. Science 2006, 312, 1191. doi: 10.1126/science. 1125925

(17) Park, S.; Ruoff, R. S. Nat. Nanotechnol. 2009, 4, 217. doi: $10.1038 /$ nnano. 2009.58

(18) Li, X. S.; Cai, W. W.; An, J. H.; Kim, S.; Nah, J.; Yang, D. X.; Piner, R.; Velamakanni, A.; Jung, I.; Tutuc, E.; et al. Science 2009, 324, 1312. doi: $10.1126 /$ science. 1171245

(19) Luong, D. X.; Bets, K. V.; Algozeeb, W. A.; Stanford, M. G.; Kittrell, C.; Chen, W. Y.; Salvatierra, R. V.; Ren, M. Q.; McHugh, E. A.; Advincula, P. A.; et al. Nature 2020, 577, 647. doi: $10.1038 / \mathrm{s} 41586-020-1938-0$

(20) Lin, L.; Deng, B.; Sun, J. Y.; Peng, H. L.; Liu, Z. F. Chem. Rev. 2018, 118, 9281. doi: 10.1021/acs.chemrev.8b00325

(21) Fiori, G.; Bonaccorso, F.; Iannaccone, G.; Palacios, T.; Neumaier, D.; Seabaugh, A.; Banerjee, S. K.; Colombo, L. Nat. Nanotechnol. 2014, 9, 768. doi: 10.1038/nnano.2014.207

(22) Hamilton, J. C.; Blakely, J. M. Surf. Sci. 1980, 91, 199. doi: 10.1016/0039-6028(80)90080-1

(23) Coraux, J.; N'Diaye, A. T.; Busse, C.; Michely, T. Nano Lett. 2008, 8, 565. doi: $10.1021 / \mathrm{n} 10728874$

(24) Sutter, P. W.; Flege, J.-I.; Sutter, E. A. Nat. Mater. 2008, 7, 406. doi: $10.1038 / \mathrm{nmat} 2166$

(25) Kwon, S.-Y.; Ciobanu, C. V.; Petrova, V.; Shenoy, V. B.; Bareno, J.; Gambin, V.; Petrov, I.; Kodambaka, S. Nano Lett. 2009, 9, 3985. doi: $10.1021 / \mathrm{nl} 902140 \mathrm{j}$

(26) Reina, A.; Jia, X. T.; Ho, J.; Nezich, D.; Son, H.; Bulovic, V.; Dresselhaus, M. S.; Kong, J. Nano Lett. 2009, 9, 30. doi: $10.1021 / \mathrm{n} 1801827 \mathrm{v}$

(27) Wei, D. C.; Liu, Y. Q.; Wang, Y.; Zhang, H. L.; Huang, L. P.; Yu, G. Nano Lett. 2009, 9, 1752. doi: 10.1021/n1803279t

(28) Gao, T.; Xie, S. B.; Gao, Y. B.; Liu, M. X.; Chen, Y. B.; Zhang, Y. F.; Liu, Z. F. ACS Nano 2011, 5, 9194. doi: 10.1021/nn203440r

(29) Gao, L. B.; Ren, W. C.; Xu, H. L.; Jin, L.; Wang, Z. X.; Ma, T.; Ma, L.-P.; Zhang, Z. Y.; Fu, Q.; Peng, L.-M.; et al. Nat. Commun. 2012, 3, 699. doi: $10.1038 /$ ncomms 1702

(30) Edwards, R. S.; Coleman, K. S. Acc. Chem. Res. 2013, 46, 23. doi: 10.1021/ar3001266

(31) Teng, P.-Y.; Lu, C.-C.; Akiyama-Hasegawa, K.; Lin, Y.-C.; Yeh, C.-H.; Suenaga, K.; Chiu, P.-W. Nano Lett. 2012, 12, 1379. doi: $10.1021 / \mathrm{nl} 204024 \mathrm{k}$

(32) Lin, L.; Zhang, J. C.; Su, H. S.; Li, J. Y.; Sun, L. Z.; Wang, Z. H.; Xu, F.; Liu, C.; Lopatin, S.; Zhu, Y. H.; et al. Nat. Commun. 2019, 10, 1912. doi: 10.1038/s41467-019-09565-4

(33) Yan, K.; Peng, H. L.; Zhou, Y.; Li, H.; Liu, Z. F. Nano Lett. 2011, 11, 1106. doi: $10.1021 / \mathrm{nl} 104000 \mathrm{~b}$

(34) Wu, T. R.; Zhang, X. F.; Yuan, Q. H.; Xue, J. C.; Lu, G. Y.; Liu, Z. H.; Wang, H. S.; Wang, H. M.; Ding, F.; Yu, Q. K.; et al. Nat. Mater. 2016, 15, 43. doi: 10.1038/nmat4477

(35) Tang, S. J.; Wang, H. M.; Wang, H. S.; Sun, Q. J.; Zhang, X. Y.; 
Cong, C. X.; Xie, H.; Liu, X. Y.; Zhou, X. H.; Huang, F. Q.; et al. Nat. Commun. 2015, 6, 6499. doi: 10.1038/ncomms 7499

(36) Bhaviripudi, S.; Jia, X. T.; Dresselhaus, M. S.; Kong, J. Nano Lett. 2010, 10, 4128. doi: 10.1021/nl102355e

(37) Qing, F. Z.; Jia, R. T.; Li, B.-W.; Liu, C. L.; Li, C. Z.; Peng, B.; Deng, L. J.; Zhang, W. L.; Li, Y. R.; Ruoff, R. S.; et al. 2D Mater. 2017, 4, 025089. doi: 10.1088/2053-1583/aa6da5

(38) Li, X. S.; Cai, W. W.; Colombo, L.; Ruoff, R. S. Nano Lett. 2009, 9 , 4268. doi: 10.1021/n1902515k

(39) Ago, H.; Ito, Y.; Mizuta, N.; Yoshida, K.; Hu, B. S.; Orofeo, C. M.; Tsuji, M.; Ikeda, K.-I.; Mizuno, S. ACS Nano 2010, 4, 7407. doi: $10.1021 / \mathrm{nn} 102519 \mathrm{~b}$

(40) Ismach, A.; Druzgalski, C.; Penwell, S.; Schwartzberg, A.; Zheng, M.; Javey, A.; Bokor, J.; Zhang, Y. G. Nano Lett. 2010, 10, 1542. doi: $10.1021 / \mathrm{n} 19037714$

(41) Seah, C.-M.; Chai, S.-P.; Mohamed, A. R. Carbon 2014, 70, 1. doi: 10.1016/j.carbon.2013.12.073

(42) Chen, J. Y.; Wen, Y. G.; Guo, Y. L.; Wu, B.; Huang, L. P; Xue, Y. Z.; Geng, D. C.; Wang, D.; Yu, G.; Liu, Y. Q. J. Am. Chem. Soc. 2011, 133, 17548. doi: 10.1021/ja2063633

(43) Li, P.; Li, Z. Y.; Yang, J. L. J. Phys. Chem. C 2017, 121, 25949. doi: 10.1021/acs.jpcc.7b09622

(44) Tan, H.; Wang, D. G.; Guo, Y. B. Coatings 2018, 8, 40. doi: $10.3390 /$ coatings 8010040

(45) Shu, H.; Tao, X.-M.; Ding, F. Nanoscale 2015, 7, 1627. doi: $10.1039 / \mathrm{c} 4 \mathrm{nr} 05590 \mathrm{j}$

(46) Wang, X. L.; Yuan, Q. H.; Li, J.; Ding, F. Nanoscale 2017, 9, 11584. doi: $10.1039 / \mathrm{c} 7 \mathrm{nr} 02743 \mathrm{e}$

(47) Deng, B.; Liu, Z. F.; Peng, H. L. Adv. Mater. 2019, 31, 1800996. doi: 10.1002/adma.201800996

(48) Gao, J. F.; Yip, J.; Zhao, J. J.; Yakobson, B. I.; Ding, F. J. Am. Chem. Soc. 2011, 133, 5009. doi: 10.1021/ja110927p

(49) Lin, L.; Sun, L. Z.; Zhang, J. C.; Sun, J. Y.; Koh, A. L.; Peng, H. L.; Liu, Z. F. Adv. Mater. 2016, 28, 4671. doi: 10.1002/adma.201600403

(50) Zhang, X. Y.; Wang, L.; Xin, J.; Yakobson, B. I.; Ding, F. J. Am. Chem. Soc. 2014, 136, 3040. doi: 10.1021/ja405499x

(51) Li, X. S.; Magnuson, C. W.; Venugopal, A.; An, J.; Suk, J. W.; Han, B. Y.; Borysiak, M.; Cai, W. W.; Velamakanni, A.; Zhu, Y. W.; et al. Nano Lett. 2010, 10, 4328. doi: 10.1021/nl101629g

(52) Sun, L. Z.; Lin, L.; Zhang, J. C.; Wang, H.; Peng, H. L.; Liu, Z. F. Nano Res. 2016, 10, 355. doi: 10.1007/s12274-016-1297-1

(53) Artyukhov, V. I.; Liu, Y. Y.; Yakobson, B. I. Proc. Natl. Acad. Sci. U. S. A. 2012, 109, 15136. doi: 10.1073/pnas.1207519109

(54) Chen, Z. L.; Qi, Y.; Chen, X. D.; Zhang, Y. F.; Liu, Z. F. Adv. Mater. 2019, 31, 1803639. doi: 10.1002/adma.201803639

(55) Jia, K. C.; Zhang, J. C.; Lin, L.; Li, Z. Z.; Gao, J.; Sun, L. Z.; Xue, R.
W.; Li, J. Y.; Kang, N.; Luo, Z. T.; et al. J. Am. Chem. Soc. 2019, 141, 7670. doi: $10.1021 /$ jacs.9b02068

(56) Köhler, C.; Hajnal, Z.; Deák, P.; Frauenheim, T.; Suhai, S. Phys. Rev. B 2001, 64, 085333. doi: 10.1103/PhysRevB.64.085333

(57) Chen, X. D.; Chen, Z. L.; Sun, J. Y.; Zhang, Y. F.; Liu, Z. F. Acta Phys. -Chim. Sin. 2016, 32, 14. [陈旭东, 陈召龙, 孙靖宇, 张艳峰, 刘忠范. 物理化学学报, 2016, 32, 14.] doi: 10.3866/PKU.WHXB201511133

(58) Wang, H. Controllable Method of Graphene Growth on Copper Foil by Chemical Vapor Deposition. Ph.D. Dissertation, Peking University, Beijing, 2015. [王欢. 石墨烯在铜箔表面上的控制生长 方法研究[D]. 北京: 北京大学, 2015.]

(59) Wang, H. P.; Xue, X. D.; Jiang, Q. Q.; Wang, Y. L.; Geng, D. C.; Cai, L.; Wang, L. P.; Xu, Z. P.; Yu, G. J. Am. Chem. Soc. 2019, 141, 11004. doi: $10.1021 /$ jacs.9b05705

(60) Chen, S. S.; Ji, H. X.; Chou, H.; Li, Q. Y.; Li, H. Y.; Suk, J. W.; Piner, R.; Liao, L.; Cai, W. W.; Ruoff, R. S. Adv. Mater. 2013, 25, 2062. doi: 10.1002/adma.201204000

(61) Muñoz, R.; Gómez-Aleixandre, C. Chem. Vapor Depos. 2013, 19, 297. doi: $10.1002 /$ cvde.201300051

(62) Hu, C. X.; Li, H. J.; Zhang, S. Y.; Li, W. J. Mater. Sci. 2016, 51, 3897. doi: 10.1007/s10853-015-9709-2

(63) Li, Z. C.; Zhang, W. H.; Fan, X. D.; Wu, P.; Zeng, C. G.; Li, Z. Y.; Zhai, X. F.; Yang, J. L.; Hou, J. G. J. Phys. Chem. C 2012, 116, 10557. doi: $10.1021 / j p 210814 j$

(64) Lewis, A. M.; Derby, B.; Kinloch, I. A. ACS Nano 2013, 7, 3104. doi: $10.1021 / \mathrm{nn} 305223 \mathrm{y}$

(65) Li, G.; Huang, S. H.; Li, Z. Y. Phys. Chem. Chem. Phys. 2015, 17, 22832. doi: $10.1039 / \mathrm{c} 5 \mathrm{cp} 02301 \mathrm{~g}$

(66) Shivayogimath, A.; Mackenzie, D.; Luo, B. R.; Hansen, O.; Boggild, P.; Booth, T. J. Sci. Rep. 2017, 7, 6183. doi: 10.1038/s41598-017-06276-y

(67) Öberg, H.; Nestsiarenka, Y.; Matsuda, A.; Gladh, J.; Hansson, T.; Pettersson, L. G. M.; Öström, H. J. Phys. Chem. C 2012, 116, 9550. doi: $10.1021 / j p 300514 \mathrm{f}$

(68) Hao, Y. F.; Bharathi, M. S.; Wang, L.; Liu, Y. Y.; Chen, H.; Nie, S.; Wang, X. H.; Chou, H.; Tan, C.; Fallahazad, B.; et al. Science 2013, 342, 720. doi: 10.1126/science.1243879

(69) Safron, N. S.; Arnold, M. S. J. Mater. Chem. C 2014, $2,744$. doi: $10.1039 / \mathrm{c} 3 \mathrm{tc} 31738 \mathrm{~b}$

(70) Xu, X. Z.; Zhang, Z. H.; Qiu, L.; Zhuang, J. N.; Zhang, L.; Wang, H.; Liao, C. N.; Song, H. D.; Qiao, R. X.; Gao, P.; et al. Nat. Nanotechnol. 2016, 11, 930. doi: 10.1038/nnano.2016.132

(71) Liu, C.; Xu, X. Z.; Qiu, L.; Wu, M. H.; Qiao, R. X.; Wang, L.; Wang, J. H.; Niu, J. J.; Liang, J.; Zhou, X.; et al. Nat. Chem. 2019, 11, 730. doi: 10.1038/s41557-019-0290-1

(72) Li, Q. C.; Zhao, Z. F.; Yan, B. M.; Song, X. J.; Zhang, Z. P.; Li, J.; 
Wu, X. S.; Bian, Z. Q.; Zou, X. L.; Zhang, Y. F.; et al. Adv. Mater. 2017, 29, 1701325. doi: 10.1002/adma.201701325

(73) Yazyev, O. V.; Louie, S. G. Nat. Mater. 2010, 9, 806. doi: $10.1038 /$ nmat 2830

(74) Pop, E.; Varshney, V.; Roy, A. K. MRS Bull. 2012, 37, 1273. doi: $10.1557 / \mathrm{mrs} .2012 .203$

(75) Karoui, S.; Amara, H.; Bichara, C.; Ducastelle, F. ACS Nano 2010, 4, 6114. doi: $10.1021 / \mathrm{nn} 101822 \mathrm{~s}$

(76) Zan, R.; Ramasse, Q. M.; Bangert, U.; Novoselov, K. S. Nano Lett. 2012, 12, 3936. doi: 10.1021/n1300985q

(77) Rümmeli, M. H.; Gorantla, S.; Bachmatiuk, A.; Phieler, J.; Geißler, N.; Ibrahim, I.; Pang, J.; Eckert, J. Chem. Mater. 2013, 25, 4861. doi: $10.1021 / \mathrm{cm} 401669 \mathrm{k}$

(78) Sun, J. Y.; Chen, Y. B.; Priydarshi, M. K.; Chen, Z.; Bachmatiuk, A.; Zou, Z. Y.; Chen, Z. L.; Song, X. J.; Gao, Y. F.; Rummeli, M. H.; et al. Nano Lett. 2015, 15, 5846. doi: 10.1021/acs.nanolett.5b01936

(79) Chen, Z. L.; Qi, Y.; Chen, X. D.; Zhang, Y. F.; Liu, Z. F. Adv. Mater. 2019, 31, 1803639. doi: 10.1002/adma.201803639

(80) Zhang, J. C.; Sun, L. Z.; Jia, K. C.; Liu, X. T.; Cheng, T.; Peng, H. L.; Lin, L.; Liu, Z. F. ACS Nano 2020, 14, 10796. doi: 10.1021/acsnano.0c06141

(81) Zhang, J. C.; Jia, K. C.; Lin, L.; Zhao, W.; Quang, H. T.; Sun, L. Z.; Li, T. R.; Li, Z. Z.; Liu, X. T.; Zheng, L. M.; et al. Angew. Chem. Int. Ed. Engl. 2019, 58, 14446. doi: 10.1002/anie.201905672

(82) Sun, L. Z.; Lin, L.; Wang, Z. H.; Rui, D. R.; Yu, Z. W.; Zhang, J. C.; Li, Y. L. Z.; Liu, X. T.; Jia, K. C.; Wang, K. X.; et al. Adv. Mater. 2019, 31, 1902978. doi: 10.1002/adma.201902978

(83) Jia, K. C.; Ci, H. N.; Zhang, J. C.; Sun, Z. T.; Ma, Z. T.; Zhu, Y. S.; Liu, S. N.; Liu, J. L.; Sun, L. Z.; Liu, X. T.; et al. Angew. Chem. Int. Ed. Engl. 2020, 59, 17214. doi: 10.1002/anie.202005406

(84) Liu, X. T.; Zhang, J. C.; Chen, H.; Liu, Z. F. Acta Phys. -Chim. Sin. 2022, 38, 2012047. [刘晓婷, 张金灿, 陈恒, 刘忠范. 物理化学学 报, 2022, 38, 2012047.] doi: 10.3866/PKU.WHXB202012047

(85) Xie, H. H.; Cui, K. J.; Cui, L. Z.; Liu, B. Z.; Yu, Y.; Tan, C. W.; Zhang, Y. Y.; Zhang, Y. F.; Liu, Z. F. Small 2020, 16, 1905485. doi: $10.1002 / \mathrm{smll} .201905485$

(86) Chen, X. D.; Chen, Z. L.; Jiang, W. S.; Zhang, C.; Sun, J. Y.; Wang, H. H.; Xin, W.; Lin, L.; Priydarshi, M. K.; Yang, H.; et al. Adv. Mater. 2017, 29, 1603428. doi: 10.1002/adma.201603428

(87) Castro Neto, A. H.; Guinea, F.; Peres, N. M. R.; Novoselov, K. S.; Geim, A. K. Rev. Mod. Phys. 2009, 81, 109. doi: 10.1103/RevModPhys.81.109

(88) Ta, H. Q.; Perello, D. J.; Duong, D. L.; Han, G. H.; Gorantla, S.; Nguyen, V. L.; Bachmatiuk, A.; Rotkin, S. V.; Lee, Y. H.; Rummeli, M. H. Nano Lett. 2016, 16, 6403. doi: 10.1021/acs.nanolett.6b02826
(89) Deng, B.; Xin, Z. W.; Xue, R. W.; Zhang, S. S.; Xu, X. Z.; Gao, J.; Tang, J. L.; Qi, Y.; Wang, Y. N.; Zhao, Y.; et al. Sci. Bull. 2019, 64, 659. doi: 10.1016/j.scib.2019.04.030

(90) Huet, B.; Zhang, X.; Redwing, J. M.; Snyder, D. W.; Raskin, J.-P. 2D Mater. 2019, 6, 045032. doi: 10.1088/2053-1583/ab33ae

(91) Jiang, B.; Zhao, Q. Y.; Zhang, Z. P.; Liu, B. Z.; Shan, J. Y.; Zhao, L.; Rümmeli, M. H.; Gao, X.; Zhang, Y. F.; Yu, T. J.; et al. Nano Res. 2020, 13, 1564. doi: 10.1007/s12274-020-2771-3

(92) Huang, P. Y.; Ruiz-Vargas, C. S.; van der Zande, A. M.; Whitney, W. S.; Levendorf, M. P.; Kevek, J. W.; Garg, S.; Alden, J. S.; Hustedt, C. J.; Zhu, Y.; et al. Nature 2011, 469, 389. doi: $10.1038 /$ nature 09718

(93) Cui, T.; Mukherjee, S.; Sudeep, P. M.; Colas, G.; Najafi, F.; Tam, J.; Ajayan, P. M.; Singh, C. V.; Sun, Y.; Filleter, T. Nat. Mater. 2020, 19, 405. doi: 10.1038/s41563-019-0586-y

(94) Ma, T.; Liu, Z. B.; Wen, J. X.; Gao, Y.; Ren, X. B.; Chen, H. J.; Jin, C. H.; Ma, X. L.; Xu, N. S.; Cheng, H. M.; et al. Nat. Commun. 2017, 8, 14486. doi: 10.1038/ncomms 14486

(95) Lin, L.; Li, J. Y.; Ren, H. Y.; Koh, A. L.; Kang, N.; Peng, H. L.; Xu, H. Q.; Liu, Z. F. ACS Nano 2016, 10, 2922. doi: 10.1021/acsnano.6b00041

(96) Vlassiouk, I. V.; Stehle, Y.; Pudasaini, P. R.; Unocic, R. R.; Rack, P. D.; Baddorf, A. P.; Ivanov, I. N.; Lavrik, N. V.; List, F.; Gupta, N.; et al. Nat. Mater. 2018, 17, 318. doi: 10.1038/s41563-018-0019-3

(97) Wang, H.; Xu, X. Z.; Li, J. Y.; Lin, L.; Sun, L. Z.; Sun, X.; Zhao, S. L.; Tan, C. W.; Chen, C.; Dang, W. H.; et al. Adv. Mater. 2016, 28, 8968. doi: 10.1002/adma.201603579

(98) Sun, X.; Lin, L.; Sun, L. Z.; Zhang, J. C.; Rui, D. R.; Li, J. Y.; Wang, M. Z.; Tan, C. W.; Kang, N.; Wei, D.; et al. Small 2018, 14, 1702916. doi: 10.1002/smll.201702916

(99) Xie, Y. D.; Cheng, T.; Liu, C.; Chen, K.; Cheng, Y.; Chen, Z. L.; Qiu, L.; Cui, G.; Yu, Y.; Cui, L. Z.; et al. ACS Nano 2019, 13, 10272. doi: 10.1021/acsnano.9b03596

(100) Xu, J. B.; Hu, J. X.; Li, Q.; Wang, R. B.; Li, W. W.; Guo, Y. F.; Zhu, Y. B.; Liu, F. K.; Ullah, Z.; Dong, G. C.; et al. Small 2017, 13, 1700651. doi: 10.1002/smll.201700651

(101) Zhang, Y. N.; Huang, D. P.; Duan, Y. W.; Chen, H.; Tang, L. L.; Shi, M. Q.; Li, Z. C.; Shi, H. F. Nanotechnology 2020, 32, 105603. doi: 10.1088/1361-6528/abcceb

(102) Chen, Z. L.; Guan, B. L.; Chen, X.-D.; Zeng, Q.; Lin, L.; Wang, R. Y.; Priydarshi, M. K.; Sun, J. Y.; Zhang, Z. P.; Wei, T. B.; et al. Nano Res. 2016, 9, 3048. doi: 10.1007/s12274-016-1187-6

(103) Liu, B. Z.; Sun, J. Y.; Liu, Z. F. ChemNanoMat 2020, 6, 483. doi: 10.1002/cnma.202000045 